\title{
Fin Fall von Mikrophthalmus congenitus unilateralis
}

nebst einigen Bemerkungen über die vermuthliche Aetiologie und Entwicklungsgeschichte desselben.

Von

Dr. Hermann Becker.

(Aus dem Laboratorium der Univers.-Augenklinik in München.) Hierzu Tafel IV, Fig. 1-5.

\section{Einleitung.}

In der Teratologie des Auges hat das Capitel, welches die Missbildungen behandelt, die den ganzen Bulbus betreffen (Anophthalmus, Mikrophthalmus etc.), in den letzten Decennien zwar eine grosse Bereicherung erfahren, jedoch ist die Zahl der mikroskopisch untersuchten Fälle eine ziemlich kleine, und die Beobachter beschränken sich meistens auf eine äusserliche Beschreibung. Der vorliegende Fall dürfte desshalb von grösserem Interesse sein, weil die Entwicklungsstörung in den Beginn des zweiten Actes der Ophthalmogense (Manz) fällt, also die Bildung der secundären Augenblase and der Linse betrifft.

\section{Pathologisch-anatomischer Befund.}

Das Kind, ein Mädchen, wurde in der Frauenklinik zu München geboren. Gewicht 2600 gr. Länge $45 \mathrm{~cm}$. Geburtsverlauf normal; die Fruchthüllen zeigten keine Anomalien, keine amniotischen Verwachsungen. Die Augen der Eltern sind gesund. 


\section{A. Makroskopischer Befund.}

I. Am 1 linat alten Kinde.

Der Körper des Kindes ist gut entwickelt. An Schädel fallen etwas breite Fontanellen auf; die Stime ist symmetrisch entwickelt, nur die linke Glabella erscheint etwas flacher. Augenbrauen gleichmässig gut entwickelt. Der Supraorbitalrand tritt links nicht so deutlich hervor wie rechts und $z$ war sind die ausseren zwei Drittel abgeflacht. Es fehlt auch links das subcutane Fettpolster, welehes um das rechte Auge, wie auch am ganzen übrigen Körper gut entwickelt ist. An den Nasen ist nichts besonderes wahrzunehmen. Die Lider des rechten Auges sind völlig normal; links dagegen sind sie, anstatt rom Bulbus vorgewölht zu sein, trichterförmig eingesunken, und zwar das obere mehr als das untere. Die Lidspalte ist links, da das Kind die Lider nicht bewegen kann, immer geschlossen und beträgt nur zwei Drittel von der des rechten Auges. Am Oberlide fehlt der sulcus palpebralis superior, da die Haut sich rom Supraorbitalrand glatt auf das eingesunkene Lid hinüberzieht. Das untere Lid sehiebt sich etwas vor das obere, weshalb die Cilien des oberen nach oben gerichtet stehen. Die Wimpern sind an beiden Lidern in reichlicher Anzahl vorhanden, nur etwas kleiner als rechts. Die Lidspalte ist nicht verwachsen, die Thränenpunkte und die Caruncula lacrymalis sind vorhanden, es fehlt jedoch eine Plica semilunaris.

Zieht man die Lider vorsichtig anseinander, so sieht man in einen leeren Raum, an dessen hinterem Ende (Stelle des Opticuseintrittes) eine porcellanweisse Prominenz von $2 \mathrm{~mm}$ Durchmesser sich findet. Der ganze Raum wird von einer Schleimhaut ausgekleidet, welche auch die Prominenz überzieht, woselbst sie keine Gefässe besitzt. Bei Bewegungen des rechten Auges bewegt sich auch die Prominenz und zwar meistens coordinirt.

Vor beiden Ohrmuscheln sitzen am Antitragus kleine gestielte Auswüchse, offenbar fibröser Natur. Die Thränensecretion war auf beiden Augen gleichmässig stark.

Während des Wachsthums in den folgenden 4 Monaten offnete sich die Lidspalte, sodass man frei in den Conjunctivalsack hineinsehen konnte. Das Knochenwachsthum war offenbar ein gutes, während die linken Lider in der Weiterentwicklung zurückblieben. Schon als ich im 3. Monat zu dem erkrankten 
Kinde gerufen wurde, war ich erstaunt über den Wachsthumsfortschritt der kleinen weissen Prominenz, welche jetzt einen offenbaren Mikrophthalmus diagnosticiren liess, welcher mit dem anderen Bulbus coordinirt bewegt wurde.

Das Kind starb im Beginn des 6. Monates an Magendarmcatarrh. Die Section der Leiche wurde von mir 14 Stunden nach dem Tode gemacht.

II. Makroskopischer Befund an der Leiche.

Organe der Brusthöble: Offenbleiben des Foramen ovale; die linke Lunge besass vollständige 3 Lappen, die rechte eine Doppellappung des Mittellappens. Gefässanomalien waren nicht vorhanden.

Organe der Bauchhöhle: normal.

Kopf: andere Hissbildungen, wie sie sonst häufig bei Anophthalmus beobachtet worden sind, sind nicht vorhanden. An der Schädelbasis fällt nur eine Abflachung des linken Orbitaldaches auf; eine Verkürzung der Basis cranii war nicht vorhanden. Die Präparation der rechten Augenhöhle ergab normale Verhältnisse. Links lag der Nervus supraorbitalis trigemini zu weit lateral, und ebenso verlief der Nervus frontalis durch ein ganz lateral gelegenes Foramen zur Stime. Alle Augenmuskeln waren vorhanden und nur nicht so kräftig entwickelt wie rechts; besonders gilt dies von dem äusserst minimalen Levator palpebrae. Es fehlte ferner keiner der durch die Orbita verlaufenden Nerven, welche alle bis auf den Opticus eine gute Entwicking zeigten. Der Opticus war im Wesentlichen dünner als der rechte (um $1 / 3$ ). Die Arteria ophthalmica war ein wenig dünner als die rechte, und es fand sich keine Arteria centralis retinae, welche an der lateralen Seite des Optieus eintrat; dagegen verliefen Gefässe zur untern Seite des Opticuseintritts in den Mikrophthalmus und traten hier in denselben ein.

Verticaler Durchmesser:
R. Bulbus: $18 \mathrm{~mm}$.
L. Bulbus: $9,5 \mathrm{~mm}$.

Sagittaler Durchmesser:

R. Bulbus: $18,7 \mathrm{~mm}$. L. Bulbus: $8,0 \mathrm{~mm}$.

Transversaler Durchmesser:
R. Bulbus: $18,5 \mathrm{~mm}$.
L. Bulbus: $0,9 \mathrm{~mm}$. 
Aus diesen Nessungen ergiebt sich fïr den lileinen Bulbus die Gestalt eines von vorne nach hinten, also in sagittaler Richtung abgeplatteten Ellipsoids. Er ist dem rechten Augapfel gegenüber im verticalen und transversalen Durchmesser fast auf die Hälfte reducirt und im sagittalen auf noch etwas mehr als die Hälfte.

Nach C. Krause*) soll, wie dies ja auch beim reehten Bulbus der Fall ist, der verticale Durchmesser etwas kleiner sein als die beiden anderen. Beim kleinen Bulbus ist dies nun nicht der Fall, sondern der sagittale Diameter bleibt um $1-1,5 \mathrm{~mm}$ hinter den beiden anderen zuräck. Die Ursache dieser Abplattung werden wir später kennen lernen.

Eine Abgrenzung der Cornea von der Sclera, einen Limbus corneae, konnte ich nicht erkennen; die Hornhant war weissbläulich und undurchsichtig. An normaler Stelle lag links eine etwas kleinere Thränendrüse als rechts. Da links die formative Wirkung des Augapfels wegen der Kleinheit desselben und wegen seiner Lage tief hinten in der Orbita sich nicht auf die Lider hatte geltend machen hönnen, so zeigen diese eine einfach platte bintere Fläche, und ebenso fehlen die Fornices conjunctivae, da die Schleimhant ohne Faltung von Lidern in den Conjunctivalsack äbergeht. Die Länge der Lidspalte beträgt 1. $13 \mathrm{~mm}$, r. $19 \mathrm{~mm}$.

An der knöchernen Orbita nahm ich folgende Maasse:

Horizontaler Durchmesser der vorderen Oeffnung:
r. $26,5 \mathrm{~mm}$.
1. $21,5 \mathrm{~mm}$.

Senkrechter Durchmesser derselben:
r. $24,2 \mathrm{~mm}$.
1. $21,0 \mathrm{~mm}$.

Tiefe der Orbita von der Mitte des Infraorbitalrandes bis zum Processus orbitalis ossis palatini:
r. $21,8 \mathrm{~mm}$.
1. $21,0 \mathrm{~mm}$.

Dass das Auge das für die Schädelgestaltung wichtigste Sinnesorgan ist, haben die Forschungen v. Gudden's **) und anderer Autoren deutlich gezeigt. Gudden fand bei Enucleation des Bulbus beim neugebornen Thiere, dass eine

*) No. 29 S. 78.

**) No. 6. 
bedeutende Verengerung der Orbita und der Schädelbasis eintrat, Die Erfahrungen, welche G. Joseph *) bei seinen Studien, „über die Gestaltung der knöchernen Augenhöhle nach Verlust des Augapfels" machte, entlehne ich einem Referat in Michel's Jahresberichten. Joseph meint, dass die Verkleinerung nur in der Höhe eintrete und mit einer Verflachung des Daches einhergehe, dass der Boden sich etwas hebe und die Augenöffnung "mehr spaltförmig in die Breite gezogen" erscheine. Nur einen Fall konnte ich in der Litteratur auffinden, wo bei Monophthalmus beide Orbitae gleich gross gewesen sein sollen. Ihn beobachtete Schenkl**) an einem drei Wochen alten Knaben. Im vorliegenden Falle haben wir nun kein Fehlen des Augapfels, sondern einen auf die Hälfte an Grösse reducirten, welcher im Wachsthum mit fortschritt. Trotzdem hat dies genügt, um gegenüber der normal ausgebildeten rechten Orbita links eine Verengerung derselben in der verticalen Richtung um $3,2 \mathrm{~mm}$ und in der horizontalen um $5,0 \mathrm{~mm}$ zu bewirken; ausserdem die Abflachung des Orbitaldaches. Ich kann somit die Erfahrungen Joseph's in Betreff des spaltförmig in die Breite Gezogenseins nicht constatiren, sondern finde in meinem Fall gerade in der horizontalen Richtung die grősste Verengerung.

Eine Verkürzung der Schädelbasis war in meinem Falle nicht vorhanden. An dem die Orbita bildenden Knochen fiel ausser der schon oben erwähnten Abflachung des Margo supraorbitalis die mangelhafte Entwicklung des Os. lacrymale auf. Die Crista trat nur wenig scharf hervor und die Oeffnung des knöchermen Ductus naso-lacrymalis war ganz bedeutend enger, als rechts. Ieh glaube, dass dies Zurückbleiben im Wachsthum, wie es beim Os. lacrymale, den Lidern, der Thränondrüse, den Augenmuskeln der Fall ist, zum grössten Theil auf einer mangelhaften Ernährung derselben beruht, welehe die Ver-

*) No. 10 .

**) No.33. 
mehrungs- vud Bildungsfähigkeit der Zellen schon in sehr früher Zeit beeinträchtigte; daher auch die kleine Arteria ophthalmica. Ein mechanisches Moment ist wohl kanm anzunehmen. Die Thränendrüse hätte ja sonst, đa ein normal wachsender Bulbus sie nicht hinderte und der leere Conjunctivalsack ihr eine Fülle an Raum bot, sich eher vergrössern müssen, als kleiner bleiben.

Der Opticus der linken Seite hatte gegenüber der weissliclien Farbe des rechten ein grauröthliches Aussehen. Die Längenmessungen vom Chiasma bis zum Eintritt in den Bulbus ergaben eine Differenz von $2,5 \mathrm{~mm}$. Zum Theil beruht dieselbe anf der Lage des kleinen Bulbus näher zum Foramen optieum hin, zum Theil darauf, dass der linke Opticus gerade gestreekt verlänft, anstatt die normale Schlängelnng innerhalb des Orbita zu zeigen. Wie der linke Opticus, so war auch die ganze linke Hälfte des Chiasma und der linke Tractus nervi optici bedeutend dünner als rechts; in etwas weniger auffallendem Grade konnte man eine geringe Entwicklung des linken Ganglion geniculatum und Thalamus opticus wahrnehmen. Gefasssanomalien an der Gehirnbasis fanden sich nicht, und ebenso waren alle übrigen Hirnnerven auf beiden Seiten in normaler Weise entwickelt. Die Grössenverhältnisse der Optici etc. werde ich später genaner an Querschnitten des gehärteten Präparates geben.

Das Präparat (Bulbi, Optici, Chiasma, Thalami, Tractus und die Lider) wurde in Müller'scher Flüssigkeit monatelang. gehärtet, dann völlig entwässert, in toto in Alanncarmin gefärbt, in Alkohol 70-80 pCt. nachgehärtet, dann in absolutem Alkohol, Nelkenöl ãă, reines Nelkenöl, Terpentin und Paraffin àã, gelegt und endlich in Paraffin eingebettet. Die Optici wurden $5 \mathrm{~mm}$ hinter der Eintrittsstelle in die Bulbi quer durchschnitten, und es wurde dann, nachdem zuvor noch der rechte Angapfel in eine vordere und hintere Hälfte getheilt war, aus den Bulbi eine Serie von horizonta'en Schnitten angefertigt, deren Dicke $1 / 30-1 / 50 \mathrm{~mm}$ beträgt. Die Optici, das Chiasma und die Tractus wurden quer geschnitten und aus der Mitte der Lider einige senkrecht zum Lidspalt gelegte Schnitte genommen.

\section{B. Mikroskopischer Befund.}

Der besseren Uebersicht und des leichteren Verständnisses wegen will ich den nachfolgenden Befund der Hauptsache nach kurz in drei Punkten zusammenfassen: 
1. Fs fehlt die Linse und jegliche Andeutung ihrer Anlage.

2. Es fehlt die Pupillaröffnung am distalen $\mathrm{P}_{0}$ ] sammt Iris, Ciliarkörper und der vorderen Augenkammer.

3. Colobom der Retina, des Pigmentepithels, der Chorioidea und Verdünnung der Sclera an dieser Stelle.

Ich will nun versuchen, eine möglichst klare Beschreibung eines durch die Augenpole gelegten Horizontalschnittes zu geben und dann, von diesem ausgehend, eine kürperliche Darstellung mit Hülfe der übrigen Serienschnitte zu lietern. Zugleich werde ich immer die einzelnen Schichten des linken Mikrophthalmus mit denen des fast normal entwickelten rechten Bulbus vergleichen und bei dieser Gelegenheit auch das wenige Abnorme des letzteren berichten.

\section{Meridionaler Horizontalschnitt.}

Die äussere Schicht des Mikrophthalmus besteht aus einer Tunica fibrosa, welche sich nicht gentigend in all' ibren Theilen differencirt hat; man findet keine schärfere Trennung zwischen Sclera und Cornea, und der Untersehied zwischen beiden besteht nur in einer mehr lamellösen Schichtung des Bindegewebes and dem Fehlen äquatorialer Faserzüge in der Hornhaut. Ferner zeichnet sich die Cornea aus durch einen grossen Reichthum an Blutgefăssen und Bindegewebskernen, durch das Fehlen der Basalmembranen und des hinteren Endothels. Das vordere Corneaepithel besitzt nur polyedrische und abgeplattete Zellen, an keiner Stelle finden sich die basalen Cylinderzellen. Thre Zellen sind in der Mitte am grössten, an der Grenze der Hornhautsubstanz dagegen viel kleiner und eng gedrängt. Die Dicke der Epithelschicht beträgt links 0,036-0,054 mm (rechts: 0,03 bis $0,04 \mathrm{~mm}$ ). Unter derselben kann $\operatorname{man}$ an einigen Stellen eine schmale, sich aufhellende Zone derHornhautsubstanz wahrnehmen, welche vielleicht eine beginnende Iasalmembranbildung andentet; doch sind diese Stellen nicht so ausgesprochen deutlich, dass man sie für die Entwicklung der Basalmembran aus 
mosodermalem Gewebe verwerthen könnte. Die Dicke der ganzen Corneaanlage beträgt links: $0,54-0,67 \mathrm{~mm}$.

Am rechten Auge zeigt die Hornhaut in der Mitte eine starke Verdünnung: 0,58 am Rande, 0,27 in der Mitte; (Schwalbe*) giebt für das Ange eines Erwachsenen 1,1:0,8 mm an) sie ist aber in allen ihren Theilen gut entwickelt. Das Epithel der Cornea des Mikrophthalmus geht in das der Schleimhaut des Conjunctivalsackes über.

Die Sclera besteht aus meridionalen und äquatorialen Bindegewebszügen mit noch grossem Kernreichthum. Ihre Dicke beträgt an der lateralen Seite des Foramen opticum: 0,45-0,5 mm, (rechts $0,72 \mathrm{~mm}$ ), am Aequator ist sie nur wenig dünner (rechts dagegen $0,36 \mathrm{~mm}$ ). Medianwärts vom Selmerveneintritt ist sie dünner und in einer Entfernung von $2,3 \mathrm{~mm}$ nur $0,24 \mathrm{~mm}$ dick. Diese Verdünnung findet auf Kosten der inneren Schichten statt (s. Fig. 1).

Die Fintrittsstelle des Sehnerven zeigt nicht, wie im rechten Auge, eine conische Verengerung, deren engste Stelle in der Höhe der Glaslamelle der Chorioidea liegt, sondern hat ihre engste Stelle am äusseren Rande der Sclera und erweitert sich dann trichterfôrmig distalwärts (s. Hig. 1). Ferner liegt das Ende des intravaginalen Raumes weiter proximalwärts, nicht im Gebiet der Sclera wie rechts, sondern ausserhalb derselben. Die Dural- and Píalscheide des Sehnerven gehen unter normalen Verhältnissen im Uebrigen in die Sclera über. Die Breite der engsten stelle des Foramen opticum in der Sclerotica beträgt links $0,57 \mathrm{~mm}$, rechts $0,63 \mathrm{~mm}$. Die Lamina cribrosa werde ich beim Sehnerven besprechen.

Die Tunica vasculosa zeigt uns eine bedentende Abweichung von den normalen Verhältnissen, denn es fehlt ihr die Iris mit der Pupille; sie ist also am distalen Pol vollständig geschlossen (s. Fig. 1). Die Chorioidea propria besitzt dieselbe histologische Structur wie im rechten Auge, nur ist sie nicht ubberall gut entwickelt. Lateral vom Sehnerven ist sie sehr breit, $0,18 \mathrm{~mm}$ (rechts ebenso); sie wird dann sehr bald dünner, ist am Aequam tor der lateralen Seite nur $0,045 \mathrm{~mm}$ dick, der medialen Seite $0,03 \mathrm{~mm}$ und hört an der obenerwähnten Stelle der seleralen Verdünnung ganz auf; erscheint auch bis zum Foramen opticum nicht wieder (s. Fig. 1 und 4). Die Chorioidea beider Augen

*3. No. 30 . 
besitzt nur sebr wenig Pigment, am meisten findet sich dasselbe noch lateral rom Sehnerven. An den dickern Stellen ist besonders stark die Suprachorioidea ausgebildet; die Glaslamelle, an diesen Stellen schon äusserst minimal arasgebildet, verschwindet an den dünnen Partien meist vollständig. Die Schicht der grossen Gefässe und die der Capillaren sind gut entwickelt. Auffällig ist die grosse Dicke der Chorioidea lateral neben dem Sehnerven $0,18 \mathrm{~mm}$. Sehwalbe*) gibt als normale Dicke im Auge eines Erwachsenen nur 0,08 $\mathrm{mm}$ an.

Nach dem vorderen Angenpol zu verdickt sich die Chorioidea und geht in ein Gewebe über, welches aus Muskelfasern, Blutgefässen und kernieichem, embryonalem Bindegewebe besteht. Dieses nimmt jenen sichelförmigen Raum ein (s. Fig. 1g), welcher an Stelle der vorderen Augenkammer hinter der Cornealanlage sich befindet und nach innen zu vom Pigmentepithel begrenzt wird. Die Richtung der Muskelfasern ist zum Theil eine meridionale, zum Theil sind dieselben quer getroffen; ausserdem finden sich noch einzelne unregelmässige Faserrichtungen. Gerade hier im Bereich der Sichel ist die Glaslamelle am besten ausgebildet, auch eine Capillarschicht ist vorhanden, Pigmentzellen fehlen. Die meridionale Ausdehnung der Sichel beträgt $3,5 \mathrm{~mm}$, ihre diekste Stelle in der Richtung der Augenaxe $0,36 \mathrm{~mm}$. Eine Andeutung eines Canalis Schlemmii, eines Fontana'schen Raumes und Ligamentum pectinatum fehlt gänzlich.

Ich komme nun zur Beschreibung der von der secundären Augenblase abstammenden Schichten, Pigmentepithel und Retina. Es zeigt sich auch hier dieselbe Erscheinung, wie bei der Tunica media, es fehlt am distalen $\mathrm{Pol}$ jegliche Spur einer Pupillaroffinung; es fehlen ferner die von der Ora serrata an distal gelegenen Theile: also die Faltung am Ciliarkörper, die einschichtige Zellenlage der sich verdünnenden Retina und dio doppelte Pigmentlage der Iris. Denken wir uns auf einem meridionalen Schnitt die Retina und das Pigmentepithel vor der Ora serrata scharf abgeschnitten und die proximalen Enden so zusammengewachsen, dass alle einzelnen Schichten genau in einander übergehen, so haben wir das Bild, welches unser horizontaler Meridionalschnitt des Mikrophthalmus uns in Bezug auf diese Schichten bietet (s. Fig. 1). Das Pigment-

*) No. 29 S. 181. 
epithel beginnt lateral vom Foramen opticum und verlăuft dann in Kreisform hinter der oben erwähnten Sichel herum zur medialen Seite, erreicht aber nun nicht das Foramen wieder, sondern hört noch etwas früher auf als die Chorioidea (s. Fig. 1 b).

Die Retina nimmt ganz denselben Verlauf, hört aber nicht wie das Pigmentepithel for dem Foramen opticum auf, sondern reicht bis an dasselbe. An jener Stelle aber, wo die Selera verdünnt ist, wo die Chorioidea und Pigmentepithelschicht fehlen, zeigt die Retina (s. Fig. 1 i) eine scheinbar ganz verworrene unregelmässige Schichtung, man kaun nur die Nervenfaserschicht, Ganglienzellenschicht und innere reticuläre Schicht deutlich ohne Unterbrechung verfolgen. Ich werde später diese Stelle einer genaueren Betrachtung unterziehen. Besonders hervorheben will ich hier noch, dass die Pigmentepithelschicht und die einzelnen Schichten der Retina vollkommen regelmasssig und ohne Unterbrechung an der distalen Peripherie, wo man doch die Pupillaröffnung erwarten müsste, verlaufen (s. Fig. 1) und man keine Spur einer Verwachsungsstelle entdecken kann.

Ehe ich mich auf die histologischen Einzelheiten dieser Schichten einlasse, will ich kurz das Abnorme des rechten Augapfels berichten. Die Pigmentlagen der Iris umfassen nicht das Irisgewobe am Pupillarrand, sondern hören $0,2 \mathrm{~mm}$ vorher auf. Ferner fehlt die Macula lutea mit der Fovea centralis und endlich zeigt die Retina Faltungen. Eine auf Meridionalschnitten birnenförmig aussehende Falte liegt unmittelbar vor der Ora serrata, eine kleine Falte befindet sich median vom Sehnerveneintritt, eine etwas oberhalb desselben (erscheint also auf Horizontalschnitten aus der oberen Hälfte des Bulbus). Der wichtigste Faltungsbezirk befindet sich an Stelle der fehlenden Macula lutea; hier sind die Faltungen auf manchen Schnitten fast unentwirrbar. Ein glücklicher Zufall hat mir jedoch einen Schnitt aus der Mitte dieser Stelle verschafft, dessen Richtung eine so günstige war, dass man leicht alle Schichten der Retina verfolgen kann. Ich komme später auf diese Faltungen wieder zurück.

Die einzelnen Zellen der Pigmentepithelsehicht des Mikrophthalmus zeigen in jeder Beziehung eine normale Beschaffenheit. Die Pigmentanhäufung ist in der distalen Hälfte etwas stärker als in der proximalen; nur die wimperartigen Fortsätze sind 
sebr spärlich vorhanden. An jener Stelle, wo die Pigmentzellen aufhören, sieht man, dass eine kleine Strecke weit pigmentlose platte Zellen vorhanden sind, welche bald cubisch werden und dann in die Körnerschicht der Retina sich verlieren. Dass ein Zusammenhang zwischen beiden vorbanden ist, werden wir auf anderen Schnitten noch deutlicher sehen.

Die Retina besitzt lateral vom Foramen opticum keine Macula lutea, sie faltet sich aber nicht wie im rechten Auge an dieser Stelle, wie Fig. 1 deutlich zeigt. Die Nervenfaserschicht ist nach beiden Seiten rom Foramen aus ziemlich gleichmässig entwickelt und nimmt nach dem distalen Pol zu allmählich ab. Sie besitzt Blutgefüsse in vormaler Anzahl und Grösse, Gliazellen und besonders in der distalen Hälfte zahlreiche Müller'sche Radialfaserkegel. Thre Dicke beträgt neben dem Foramen opticum $0,1 \mathrm{~mm}$ (rechts $0,2 \mathrm{~mm}$ ) und im distalen Abschnitt $0,025-0,03 \mathrm{~mm}$. Auf einem Horizontalschnitt des rechten Auges, welcher ein wenig unterhalb der Arteria centralis nervi optici liegt, reicht von der obenerwähnten Faltung der Netzhaut an Stelle der fehlenden Macula lutea eine Falte bis an die Papille des Sehnerven, und man sieht nun, dass die Optiensfasern von der Papille direct zur Ganglienzellenschicht der Falte verlaufen. Dies ist ein Beweis dafür, dass die Faltung kein Härtungsproduct ist, sondern im Leben bestanden haben muss, da bei postmortaler Faltenbildung sich doch auch die Opticusfasern hätten falten müssen.

Die Ganglienzellenschicht (im Mikrophthalmus) besteht meistens aus einer einfachen Lage multipolarer Ganglienzellen; nur medial rom Sehnerven und lateral von ihm findet eine Vermehrung bis auf zwei Reihen statt. Im rechten Auge findet sich an der Stelle der obenerwähnten Faltung lateral vom Sehnerven eine 5-6fache Lage von Ganglienzellen.

Die innere reticuläre Schicht ist fast überall gieich dick und sieht völlig normal aus. Thre Dicke beträgt im proximalen Abschnit links $0,03-0,035 \mathrm{~mm}$ (rechts $0,035 \mathrm{~mm}$ ) und im distalen $0,025-0,03 \mathrm{~mm}$ (rechts $0,03 \mathrm{~mm}$ ), mithin ist sie fast ebenso dick wie im rechten Auge. Diese drei Schichten der Retina beginnen lateral vom Foramen opticum, verlaufen dann in Kreisform und erreichen, ohne eine Enterbrechung zu erleiden, die mediale Seite des Sehnerven wieder (s. Fig. 1). Wir werden später sehen, dass auf Schnitten aus der unteren Hälfte des Mikrophthalmus (im medialen unteren inneren v. Graefe's Archiv für Ophthalmologie, XXXIV. 3, 
Quadranten) eine Störung auftritt, welche sich auf unserem horizontalen Meridionalschnitt schon jetzt bemerkbar macht in den übrigen Schichten der Retina (s. Fig. 1 i). Die innere Körnerschicht ist in beiden Angen sebr stark entwickelt, während Schwalbe *) die normale Dicke derselben im Auge eines Erwachsenen unweit der Papille mit $35-38 \mu$ und an der Peripherie von 16-20 $\mu$ angiebt, messe ich im linken Auge neben der Papille $0,035-0,04 \mathrm{~mm}$ (rechts $0,04 \mathrm{~mm}$ ) und am distalen Pol 0,018 mm (rechts an der Peripherie 0,02-0,024 mm). Ich muss hier noch erwähnen, dass die innere Körnersehicht des Mikrophthalmus an einigen Stellen breiter wird bis anf das Doppelte $*$ ), dabei aber lückenhaft; sie erscheint wie auseinander gezogen und man findet dann am inneren Rande die einschichtige Lage der Spongioblasten fest mit der inneren reticulären Schicht verbunden; nach aussen zu folgt dann der lückenhafte Theil, welcher durchzogen wird von Nervenfasern und Müller'schen Radialfasern, und endlich folgt die gesammte Masse der Körner als äussere Grenze dieser Schicht. Ogueff mante die Erfahrung, dass bei längerer Behandlung mit Müller'scher Flüssigkeit in den granulirten Schichten grossmaschige Netzformen entstanden. Diese sind auf meinen Schnitten allerdings unverändert, auch findest sich obige Erscheinung nur an einigen Stellen und zwar nur des linken Auges. Ob nun diese Erscheinung ein Kunstproduct ist oder auf einer abnormen Entwickelung der Körnersehicht beruht, vermag ich nicht zu entscheiden. Für eine Zerrung halte ich es nicht, da die Retina an Stellen, wo sie in Folge von Zerrung gerissen ist, alle Schichten gut erhalten zeigt. Auch halte ich es nicht für wahrscheinlich, dass die Ursache auf der Härtungsmethode beruht; warum zeigt sich dann diese Erscheinung nicht überall und auch im rechten Auge? Endlich finden wir an diesen Stellen eine starke Entwicklung der Müller'schen Fasern, ja es scheinen die äusseren Köruer hier in einzelne bleine Packete getheilt $\mathrm{zu}$ sein, und ich glaube, dass die Ursache dieser Erscheinung eine übermässige Entwicklung der auusseren Faserausstrahlungen der Radialfasern ist. Die Lage der Spongioblasten deutet ja schon allein ihren innigen Zusammenhang mit der inneren reticulären Schicht an, auch

*) No. 29 S. 101 .

**) Die Messungen wurden an ganz normalen Stellen gemacht. 
konnte ich ibre innern Fortsătze oft deutlich erkennen. Nach aussen hin, wo die Untersuchung wegen jener Lucken genau gemacht werden konnte, habe ich keine Fortsätze wahrnehmen können.

Die äussere retriculäre Schicht ist in beiden Augen ron normaler Beschaffenheit und gleichmässig entwickelt. Ihre Dicke: im proximalen Absehnitt $0,012 \mathrm{~mm}$, im distalen $0,006 \mathrm{~mm}$. In der proximalen Hälfte besonders lateral vom Sehnerven ist eine äussere Faserschicht (Henle) vorhanden; im linken Auge (Mikrophthalmus) beträgt die Länge der Fasern bis zu 0,025 mm (rechts $0,03 \mathrm{~mm}$ ). Wo die äussere Faserschicht fehlt, grenzt die Schicht der Sehzellen direkt an die änssere reticuläre Schicht.

Die Schicht der Sehzellen theilt eine an den meisten Stellen gut ausgebildete Membrana limitans in zwei Abschnitte; der innere, die äussere Körnersehicht besitzt eine Dieke von $0,035 \mathrm{~mm}$ im proximalen Theile (rechts $0,04 \mathrm{~mm}$ ) und von $0,025 \mathrm{~mm}$ am distalen Pol (rechts an der Peripherie 0,03 mm). Der äussere Abschnitt, die sog. Stäbchen- und Zapfenschicht, ist im Mikrophthalmus fast ebenso gat ansgebildet, wie im rechten Ange. Die Zapfen finden sich am zahlreichsten im proximalen $\mathrm{Ab}$ schnitt und ausserdem im lateralen vorderen Quadranten; am dünnsten ist sio im vorderen medialen Quadranten. Die Dicke der Stäbchen-Zapfenschicht beträgt $0,03-0,015 \mathrm{~mm}$ (rechts: $0,035-0,015 \mathrm{~mm}$ ).

Die Papilla nervi optici besitzt im Mikrophthalmus keine Centralgefässe und keine physiologische Excavation. In unserem meridionalen Horizontalschnitt liegt eine von oben ausgehende Falte der Retina (s. Fig. 1 k) mitten vor dem sich ansbreitenden Opticus. Eine Art von physiologiseher Excavation werden wir später bei der lörperlichen Beschreibung an der unteren Seite der Sehnervenausbreitung kennen lernen. Die Lamina cribrosa liegt etwas weit proximalwärts (s. Fig. $1 \mathrm{~d}$ ); die Opticusfasern verhalten sich normal und verlieren im Gebiet derselben ihre Markscheiden. Nach Schwalbe*) sollen die Retinalschichten am Foramen opticum entweder glatt abgeschnitten aufhören oder wenigstens in der Weise zugeschärft, dass die äusserste Schicht am weitesten gegen das Foramen hin vorragt. Im rechten Ange ist dies auch der Fall, im linken Mikrophthalmus ragen an der lateralen Seite gerade die innersten Schichten am weitesten vor,

*) No. 29 S. 115. 
ja es scheint sich die ganze Retina umzubiegen, um in die Pigmentschicht überzugehen (s. Fig. 1). Noch complicirter verhalten sich die Retinaschichten auf der medialen Seite (s. Fig. 1 und Fig. 4). Hier sehen wir, wie die Opticusfasern 2wei Wege nebmen, dio einen den normalen an der Innenflache der Retina entlang, die anderen unmittelbar an der Sclera hin (s. Fig. 4 h). Zwischen beiden nun liegt Retinagewebe in einer zum Theil ganz bestimmten Anordnung, nämlich w einander umgekehrten (s. Fig. 1 und 4). Ich komme später bei der körperlichen Beschreibung auf diese Stelle genauer zurück.

Die Dicke der ganzen Retina beträgt im Uikrophthalmus; 0,28-0,12 mm, im rechten Auge: $0,4-0,14 \mathrm{~mm}$. Die grosse Differenz zwischen 0,4 und 0,28 beruht auf der grösseren. Dicke der Nervenfaserschicht im proximalen Abschnitt des rechten Auges; ohne diese ergiebt die Messung im proximalen Abschnitt: links $0,18 \mathrm{~mm}$ and rechts $0,2 \mathrm{~mm}$.

Es bleibt mir nun noch die Beschreibung des innerhalb der Retina gelegenen Inhaltes des Mikrophthalmus übrig. Hier zeigt sich nun auf unserem horizontalen Schnitt nur Glaskörper und von Linse, Linsenkapsel und Zonula ist nicht die geringste Spur zu entdecken. Letztere verhalten sich im rechten Auge völlig normal. Der Glaskörper ist, wie die Contouren desselben auf Fig. 1 zeigen, nur wenig geschrumpft und hängt am distalen Pol fest mit der Retina zusammen; in Fig. 1 sind zwei solche Stellen angedentet; man sieht hier den Glaskörper zackig ausgezogen und in dieser Zacke einen dünnen Strang von Bindegewebszellen verlaufon, welcher mit der Retina in Verbindung stebt. Ich halte diese Bindegewebsstränge, deren Zahl ziemlich gross ist, und welche sich bis mitten in den Glaskörper verfolgen lassen, für obliterirte Gefässe. Man sieht zuweilen auf angrenzenden Schnitten an denselben Stellen in der Retina ein grösseres quergetroffenes Blutgefäss, welehes offenbar früher mit jenem obliterirten Strang in Circulation gestanden hat. Begrenzt wird der Glaskörper von einem schmalen hellglänzenden Saum, einer Hyaloidea, welche immer dem Glaskörper innig anhängt und nie an Stellen, wo der Glaskörper in Folge seiner Schrumpfung sich von der Retina retrahirt hat, an letzterer haften geblieben ist. An vereinzelten Stellen auf einigen Schnitten hat sie sich auch rom Glaskörper losgelöst und ist umgeschlagen, sodass sie bandartig im Gesichtsfeld liegt. An diesen Stellen, (Flächenbetrachtung). ersebeint sie nun aus lauter 
rundlichen Gebilden zusammengesetzt; nur selten sieht man zuweilen einen Kern. Ob diese rundlichen Gebilde für eine Art von schaumigen Protoplasma zu halten sind, welches gerade so aussieht (Herr Prof. Dr. Kupiffer hatte die Güte, mich hierauf aufmerksam zu machen) oder Reste verschwindender endothelartiger Zellen sind, vermag ich nicht zu sagen, Unmittelbar unter der Hyaloidea liegen an der Peripherie des Glaskörpers einzelne grosse, durch Alauncarmin violettroth gefärbte Zellen, die sogenannten subhyaloidealen Zellen Ciaccio's*). Die Substanz des Glaskörpers erscheint mit schwacher Vergrösserung feinkörnig granulirt. Mit Seiberts stärkstem Trockensystem sieht man ein Gewirr von Fasern und viele dunkle Pünktchen. Zuweilen konnte ich auch sternförmige Zellen mit langen Fortsätzen wahrnehmen, welche im Fasergewirr nicht weiter zu verfolgen waren. Ich vermuthe daher, dass alle diese dunkleren Pünktchen, um welche man mit Bestimmtheit keine Zellconturen wahrnehmen kann, Reste zu Grunde gehender Zellen sind. Im Glaskörper des rechtem Auges finden sich ausserdem noch massenweise leucocythenartige Zellen. Sie besitzen verschiedene Grössen, von etwas vergrösserten weissen Bluthörperchen bis zu ganz kleinen rundlichen oder ovalen Zellen, und sind gerade so wie die subhyaloidealen Zellen gefürbt.

\section{Körperliche Beschreibung des Mikrophthalmus.}

Der linke Mikrophthalmus besitzt, wie schon oben erwähnt, fast die Gestalt einer Kugel. Die äussere Hülle, Tunica fibrosa ist vollständig vorhanden und hat nur eine Oeffnung, das Foramen opticum. Denken wir uns das, auf Fig. 1 zwischen den Buchstaben a a gelegene Kreissegment, welches ich als die in der Differencirung begriffene Cornealanlage beschrieben habe, um seine Axe rotirt, so haben wir ungefähr die Ausdehnung derselben abgegrenzt. Die Differencirung ist jedoch im unteren Drittel weniger fortgeschritten als in den oberen Theilen. Das Scleragewebe erleidet medial vom Sehnerveneintritt eine starke Verdünnung (s. Fig. 1), welche gerade im horizontalen Meridian am stärksten ist und nach oben und unten hin wieder abnimmt. Eine Ausbuchtung der Sclera ist nicht vorhanden; sie ist im ganzen hinteren unteren medialen Quadranten überhaupt etwas dünner als in den übrigen. In Bezug auf Opticuscanal gilt

5) No. 29 S. 136. 
dasselbe wie rom Querschnitt. Die engste Stelle liegt etwas zu weit proximalwärts, und schon im Gebiet der Sclera findet die trichterförmige Erweiterung statt. Die Richtung des Canals ist teine radiure, sondern eine von oben medial nach unten laterale. Unmittelbar unter dem Canalis opticus wird die Sclera von zahlreichen Blutgefässen durchbohrt, welche zum Theil zur Chorioidea verlaufen, zum Theil die Opticusfasern von unten her keilförmig auseinanderdrängen, um sich dann in kleinere Gefässe zu theilen und zu Retinalgefässen zu werden.

In der Tunica media finden wir schon bedeutendere Entwicklungsstörungen. Sie besitzt keine Pupillaröffnung. Jene Sichel (s. Fig. $1 \mathrm{~g}$ ) bedeckt, körperlich als Kappe gedacht, die ganze Gegend um den distalen Pol. Diese Kappe ist nun aber nicht rund abgegrenzt, wie die Cornealanlage, sondern erstreckt sich weiter über den rorderen medialen unteren Quadranten hin bis zum Aequator. Die histologische Beschaffenheit ândent sich jedoch. Um den distaien Pol herum bestand das Gewebe aus sehr kernreichen Muskelfasern, Blutgefässen und embryonalem Bindegewebe; im unteren medialen vorderen Quadranten tritt nun das letztere sehr in den Vordergrund, und es findeu sich ausser demselben nur einige Blutgefăsse. Nedialwärts vom Foramen opticum fehlt die Tunica media gänzlich, es findet sich hier ein Coloboma chorioideae. Um die Ausdehnung der versehiedenen Colobome zu reranschaulichen, labe ich dieselben auf eine Kugelflache zu zeichnen versucht, indem ich di Grössenverhalltnisse in horizontaler Richtung direct den Horizontalschuitten entnahm und in verticaler sie aus der Anzahl und Dicke derselben berechnete. Kann meine Fig. 5 demnach auch keinen Anspruch auf mathematische Genauigkeit machen, so hoffe ich doch, dass sie ihren Zweck erfüllen wird. Das Chorioidealcolobom besitzt nur eine Ausdehnung, wie sie die punktirte Linie in Fig. 5 angiebt. Am unteren Rand des Coloboms beginnt nun die Tunica media wieder, aber nicht als Chorioidea, sondern als undifferenciertes embryonales Bindegewrebe mit Blutgefässen, welches den hinteren unteren medialen Quadranten bis zum Aequator ausfült und hier mit dem des vorderen (s. oben) zusammenhängt. Auch im lateralen unteren hinteren Quadranten findet sich vom medialen Quadranten über den verticalen Meridian himüberreichend noch embryonales Bindegewebe und beginnt die eigentliche Chorioidea erst dort, wo, wie Fig. 5 zeigt, das Pigmentcolobom aufhört. Dieses embryonale, 
zum Theil von vielen Blutgefässen durchzogene Bindegewebe verdickt sich an zwoi Stellen zu einer vorspringenden Leiste (s. Fig. $2 \mathrm{a}$ und b), welche Leisten nach unten hin zu einer sich rereinigen. Diese Verdickung findet statt im Bereich des Retinalcoloboms (s. Fig. 5), wo letzteres auf dem Horizontalschnitt einheitlich wird, fliessen eben jene beiden Leisten zusammen. Fig. 2 zeigt uns ferner (und so ist es anf jedem Schnitt im Bereich des Retinalcoloboms), dass das embryonale Bindegewebe mit dem Glaskörper in Verbindung steut durch eine Art von Strängen, welche keine weitere Structur ausser einigen Kernspuren (s. Fig. 2 b) besitzen. Ich glaube, dass wir es hier mit Spuren früherer Gefässe zu thun haben. An einer stelle, Fig. 5 e, ist ein arterielles Gefäss vorhanden, welches das völlig normale Aussehen einer kleinen Arterie hat und in fast horizontaler Lage in den Glaskörper hinein verläuft. Es scheint sich dann in Capillaren aufzulösen, denn es hört als arterielles Gefäss auf, und auf den angrenzenden Schnitten findet man nur einige dünne, aus Bindegewebszellen bestehende Strünge, wie ich sie oben bei der Glaskörperretina-Verbindung beschrieben habe. Eine ausgebildete Chorioidea findet sich also nur in den Quadranten der oberen Hälfte und in den lateralen der unteren, und zwar beginnt sie erst am Rande jenes um den distalen Pol gelegenen Gewebes, welches auf dem Horizontalschnitt (Fig. 1) zwischen a-a liegt (vergleiche das oben Gesagte) und erstreckt sich bis zum Rande des Pigmentcoloboms (s. Fig. 5).

Die Pigmentepithelschicht ist überall ausserhalb ihres Coloboms, dessen Ausdebnung die schwarze Linie auf Fig. 5 reranschaulicht, vorbanden und zwar abgesehen von einer Stelle als glatte, faltenlose Kugelfläche; auf Horizontalschnitten erscheint sie also (s. Fig. 1) als ein kreisförmig verlaufender schwarzer Streifen. Am distalen Pol findet sich keine Pupillaroffnung, keine Stelle, welche eine Verwachsung andenten könnte. Im medialen hinteren unteren Quadranten anf Fig. 5 zwischen $d$ und $f$ und bis fast zum Aequator reichend faltet sich die Pigmentschicht in einer sehr starken Weise. Die Falten springen wie Ciliarkörperfalten, nach innen vor und haben auch ein ähnliches Aussehen. An ihrer dem Glaskörper zugekehrten Seite sind sie besetzt mit einer einreibigen Lage niedriger Cylinderzellen und erst dort, wo die Faltung der Pigmentschicht aufhört, sieht man, wie diese Zellen mehrsehichtig werden und in dieKörnerschicht der Retina übergehen. Am Rande des Pigmenteoloboms hängen 
sie mit den Pigmentzellen direkt zusammen, wir haben dort die Umschlagstelle der ãusseren Lamelle der secundären Augenblase in die innere. Im Innern der Pigmentepithelfalten liegt eine schmale Zone embryonales Bindegewebe.

Die Retina, am Foramen opticum beginnend, zeigt im hintern untern medialen Quadranten ein Colobom, dessen Ausdehnung uns die gezackte Linie auf Fig. 5 veranschaulicht. Sie kleidet den ganzen distalen Abschnitt ohne Unterbrechung aus, wie die Pigmentschicht (s. Fig. 1), und ist hier nur etwas dünner als im proximalen. Um den distalen Pol herum zeigt die Retina kieine Faltungen (s. Fig. 1 h). Eine ziemlich bedeutende Falte befindet sich gerade oberhalb des Sehnerveneintritts und ragt, wie Fig. $1 \mathrm{k}$ zeigt, noch bis über die Mitte der Papille vor; in ahnlicher Weise faltet sich die Retina auch im vordern untern medialen Quadranten. Alle diese Faltungen sind leicht als solche zu erkennen, und das Pigmentepithel verändert nie an diesen. Stellen seine einfach kugelflächenartige Ausbreitung. Ganz anders verhalten sich die an das Colobom angrenzenden Netzhauttheile. Abgesehen yon einer Stelle am untern medialen Rande des Coloboms (Fig. 5 d-f) ragt, wie Fig. 5 zeigt, die Retina über das Pigmentcolobom vor, und eben diese Zone, welche von aussen nicht mehr von der Pigmentschicht bedeckt wird, zeigt uns ein ganz verworrenes Bild von den einzelnen Netzhautschichten. Betrachten wir zunăchst Fig. 2, einen Horizontalschnitt, in der Hơhe von $a$ und $b$ auf Fig 5. Die beiden Hörner des Retinalcoloboms auf Fig. 5 a und $b$ zeigen sich uns and Fig. 2 als zwei Lücken in der Retina ( $a$ und b), wo sich embryonales Bindegewebe reich an Blutgefässen mit dem Glaskörper verbindet. An der medialen Seite neben $b$ sehen wir nun, wie die Pigmentepithelzellen allmählich pigmentlos werden (c), dann länglicher und später mehrschichtig, endlich zuletzt in die Körnerschicht der Retina iibergehen. Dieser Uebergang ist an der lateralen Seite neben a ebenso nur kürzer und nicht so hlar zu erkennen. Man vergleiche auch Fig. 1, auf welcher man diesen Vebergang sehr gut erkennen kann. Eine genauere Betrachtung der scheinbar ganz verworrenen Retinabezirke (Fig. 2 d, Fig. 4) zeigt, dass, weun man von der ganz durcheinander gewürfelten Mitte absieht, an der innern und äusseren seite (Fig. 2 d, Fig. 4 a) mit einer gewissen Regelmässigkeit Nervenfasern, Ganglienzellen und innere granulirte Schicht verlaufen, welche 
aber zu einander in entgegengesetzter Lage sich befinden. Auf Fig. 4 theilen sich die Opticusfasern, die eine Abtheilung zieht zur Innenfläche des Retinalgewebes, die andere an der Aussenfläche entlang direct an der Sclera hin. Am besten verfolgen kann man die mit a bezeichnete innere granulirte Schicht mit den angrenzenden Ganglienzellen, welche fast ununterbrochen in zwei grossen Zügen wie die Nervenfasern verlaufen. Nỉcht so ununterbrochen in grossen Zügen ausgeprägt, aber ebenso wie die genannten Schichten zu einander durchweg in entgegengesetzter Reihenfolge liegend, sind die übrigen Schichten zu erkennen, die innere Körnersehicht, äussere granulirte Schicht, die äussern Körner $\left(c, b_{1}, b_{2}\right)$ und die Stäbchen und Zapfenschicht. Leider kann ich auf diesem Schnitt allein nicht zeigen, was mir die Serie von Schnitten bietet; ich habe nach genauer Durchmusterung derselben die Ueberzeugung gewonnen, dass wir es hier mit einer einfachen Duplicatur der Retina zu thun haben, welche am Rande des Pigmentcoloboms beginnt und allein auf Wachsthumsverschiebung zurückzuführen ist. Die Retina schiebt sich, abgesehen von der Stelle zwischen $d$ und $f$ anf Fig. 5 über den Rand des Pigmentcoloboms vor, biegt dann am Rande ihres eigenen Coloboms um und kehrt zum Rande des Pigmenteoloboms zurück, wobei demnach die Nervenfasern nach aussen zu liegen kommen. Diese Wiedervereinigung sehen wir vorzüglich auf unsorer Zeichnung Fig. 4, wo bei $g$ jenes umgekehrt liegende Stück Retina $\left(c_{2}\right)$ mit den letzten schon etwas pigmentlos gewordenen Zellen der Pigmentepithelschicht sich vereinigt. Ueber die Ursachen der Entstehung sowie der Verworrenheit in den Schichten werde ich meine Ansicht in der Abhandlung kundgeben. Am untern medialen Rand (Fig. 5, zwischen $d$ und fi) hören beide Schichten, welche von der innern und äussern Lamelle der secundären Augenblase abstammen, zugleich auf. Wie jedoch schon oben erwähnt wurde, beginnt eine eigentliche Retina mit allen ihren Schichten erst etwas rom Colobomrande entfernt, und nur jene einschichtige der Pigmentepithelschicht aufliegende Lage niedriger Cylinderzellen sind als Abkömmlinge der innern Lamelle der secundären Augenblase und als Fortsetzung der Retina zu betrachten. Streng genommen müssten wir also anstatt Retinalcolobom Colobom der von der innern Lamelle der secundären Augenblase abstammenden Schicht sagen. 
Der Glaskörper, der Inbalt museres Mikrophthalmus zeigt überall dieselbe Beschaffenheit. Er steht im Bereich des ganzen Retinalcoloboms mit dem dort vorhandenen embryonalen Bindegewebe in Verbindung, wie ich es oben beschrieben habe und wie es Fig. 2 zeigt. Im distalen Abschnitt liegt er besonders in der untern Hälfte der Retina eng an, und man findet circa 15 Stellen, wo grössere bindegewebig obliterirte Gefässe in die Retina übergehen. Die Umhüllungsmembran des Glaskörpers, die Hyaloidea, findet sich auf jedem Schnitt dentlich ausgesprochen, es zeigt dieselbe histologische Beschaffenheit und geht im Bereich des Retinalcoloboms in das mesodernale Gewebe über. Hier gelang es mir nur an einzelnen Stellen, die endothelartigen Zellen bis direct an das mesodermale Gewebe za verfolgen, da die Membran hier meist homogen war. Zwischen dem retrahirten Glaskörper und der Retina findet man an einzelnen Stellen eine feinkörnige detritusartige Masse, welche vielleicht eine Art Zwischenkitt gebildet hat, wie ihn Löwe*) an Embryonen beobachtete.

\section{Optici, Chiasma und Tractus n. o.}

Ihre Dickenverhältnisse zeigt uns die fünffache Vergrösserung yon Fig. 3. Wir sehen, dass die linke Seite immer der rechten an Grösse bedeutend nachsteht. Auch der Thalamus opticus ersehien links kleiner als rechts. Im linken Optieus verlaufen keine Centralgefässe: Gefässe treten unmittelbar an seiner unteren Seite am distalen Ende seines Canals durch die Sclera ein, von jenem Vorsprung aus, welchen wir auf Fig. 2 a sehen. Dieser Vorsprung drängt die Opticusfasern von unten her anseinander, und daher kommt es, dass wir an der Papille keine physiologisehe Excavation in der Mitte haben, sondern, dass dieselbe nach unten offen ist und in dem unteren Drittel liegt. Beim rechten Sehnerven sind die Centralgefässe, wie Fig. 3, II a zeigt, vorbanden, treten aber etwas spät kanm $6 \mathrm{~mm}$ hinter dem Bulbus von der Seite and unten her in ihn ein.

Ein Quersehnitt dicht hinter dem Bulbus durch den Sehnerven zeigt uns, dass die Maschen des von der Pialscheide ausgehenden Bindegewebes viel enger sind, als rechts, mithin also auch die einzelnen Nervenfaserbündel. Ebenso erscheinen die einzelnen Nervenfasern kleiner, doch ist ihre Anzahl nicht

*) No. 16 S. 549 . 
wesentlich geringer. Die beiderseits runden Optici nehmen nach dem Chiasma zu eine ovale Form an mit dem grösseren Durchmesser in der horizontalen Ebene (s. Fig. 3 b). Das Bindegewebe wird immer geringer im Nervenstamme, je nather der Schnitt dem Chiasma liegt, ist links jedoch etwas stärker ansgebildet. Auch findet sich ein geringer Farbenunterschied gleich dicker Querschnitte, indem der linke etwas mehr rosa, der rechte heller aussieht. Dieser Farbenunterschied, auf Fig. 3 durch Schattirung angedentet, ist proximalwärts vom Chiasma viel grösser, nur ungekelrrt; hier ist der rechte Tractus rosa gefärbt, während der linke ganz hell erscheint. Die einzelnen Nervenfasern des rechten Tractus erscheinen undeutlich und verwaschen im Vergleich mit denen des linken, und endlich ist rechts dás Bindegewebe vermehrt. Es liess sich auf dem Querschnitt des linken Tractus keine etwas mehr rosa gefärbte Zone erkennen, und ebenso erschien der des rechten gleichnässig rosa gefärbt, keine hellere Zone.

Die linken Augenlider besitzen alle einzelnen Bestandtheile eines normalen Lides, nur viel kleiner ausgebildet. Am wenigsten in ihrer Entwicklung sind die Meibom'schen Drüsen zurückgeblieben und sind fast so gut ausgebildet als rechts.

\section{Zur Genese und Aetiologie des Mikrophthalmus.}

Die erste Frage, welche wir beim Erklärungsversuch beantworten müssen, ist: welches Stadium normaler Entwicklung ist im vorliegenden Fall noch erreicht worden, wann trat die erste Störung auf? Die Ausbildung der Retina und des Pigmentepithels zeigt uns an, dass die primäre Augenblase sich in eine secundäre umgewandelt haben muss. Denn eine Delamination in der Wandung der primären Blase mit nachfolgender Differenzirung zu Retina und Pigmentepithel anzunehmen, ist nach dem Standpunkt unserer heutigen Wissenschaft von vorne herein undenkbar. Wie sollten wir dann auch den Glaskörper erklären? Woher käme dann ferner die Lücke in der Retina und Pigmentschicht, an deren Begrenzung wir ja dentlich 
den Zusammenhang der beiden Lameilen der secundären Augenblase wahrnehmen können. Auf normale Weise kann jedoch die Umwandlung der primären Augenblase nicht erfolgt sein, da die ganze distale Hälfte unseres Mikrophthalmus in den betreffenden Schichten keine Spur einer Pupillaröffinung zeigt, ja nicht einmal eine Nahtstelle, wo wir eine Verwachsung der Ränder vernuthen könnten. Wo blieb ferner die Linse, jenes für die ganze Gestaltung des Sehorgans in der Ophthalmogenese so wichtige formative Element? Keine Spur einer Anlage derselben ist zu entdecken. Sind wir berechtigt, in unserm Falle einfach ein Ausbleiben der ectodermalen Einstülpung anzunehmen? Manz*) sagt in seiner Abhandlung rom Mikrophthalmus in Bezug auf das Pflüger'sche Bulbusrudiment: "Es ist also nicht zu bezweifeln, dass eine embryonale Linse vollständig zu Grunde gehen kann, und da die Anlage derselben von so entscheidendem Einfluss auf die Bildung des Bulbus ist, so werden wir im Falle einer Aphakia congenita um so geneigter sein, das frühere Vorhandensein eines, wenn auch unfertigen Krystallkörpers vorauszusetzen, je mehr der innere Bau und die änssere Form des Auges der Norm sich nähern." Diese Annahme nun ist gewiss in vielen Fällen vollkommen berechtigt. Trotzdem nun Nienuand bezweifeln wird, dass mein Auge der Norm viel näher steht, als das des Pflüger'schen Falles, habe ich die Ueberzeugung, dass in meinem Falle jegliche Linsenanlage unterblieben ist und möchte mich der Hoffnung hingeben, dass auch ein so berühmter Forscher, wie Manz, von seiner im Allgemeinen aufgestellten Regel hier eine Ausnahme machen wird.

Die Genese meiner Missbildung lässt sich viel einfacher und besser erklären, wenn man die Linsenanlage als ausgeblieben betrachtet; ja die Annahme einer solehen macht,

*) No. 19 S. 164 . 
wie wir sehen werden, eine Erklärung fast unmöglich. War eine ectodermale Einstülpung ursprünglich vorhanden, welche dann später gänzlicher Zerstörung anheimfiel, so bätte sich doch die primäre Augenblase am distalen Pol einstülpen müssen. $\mathrm{Manz} *$ ) und Schwalbe $*$ ) nehmen ja die Linsenbildung als Ursache für die Finstülpung der primären Augenblase an, dann würde also eine Pupillaröfnung vorhanden sein müssen, oder im Falle einer Verwachsung eine Nahtstelle am distalen Pol. Davon ist nun aber keine Spur zu finden; Retina und Pigmentepithelschicht verlaufen im ganzen distalen Abschnitt immer nebeneinander ohne jegliche Spur eines Zusammenhanges, welcher uns an die Umschlagsstelle der beiden Lamellen der secundären Augenblase erinnern könnte. Dabei hätte ja auch der Stiel der von der ectodermalen Einstülpung (Linsenanlage) mit eingestülpter Mesodermaschicht verschwinden müssen, und zwar sehr bald; denn hätte die Verwachsung erst später stattgefunden, so könnten wir, da ja die Randzone der innern Lamelle zu einer einfachen Lage von Cylinderzellen auswächst, am distalen Pol keine ausgebildete Retina vorfinden. Betrachten wir einmal das Zusammenwachsen der Ränder der secundären Augenblase, wie es ja immer an der ventralen Fläche derselben, an der sogenannten fötalen Augenspalte, stattfindet. Neuere Forschungen von Schwalbe***) und Vossius und anderen Autoren haben ergeben, dass die Macula lutea an der Stelle der Verwachsung liegt. Lassen wir jedoch diese Streitfrage ausser Acht, so zeigt uns die Lehre rom Colobom, dass ein ganz regelmässiger Ablauf des embryonalen Wachsthums erforderlich ist, um alle Spuren des Sohliessungsprocesses zu verwischen, und dass im Falle einer Störung Anomalien zuräckbleiben, wie das Fehlen des Pigments,

*) No. 17 S. 164.

No. 29 S. 78.

* No. 29 S. 83 u. 86 . 
eine abnorme Schichtung der Retinalelemente oder eine Faltung der Retina, wie im rechten Auge meines Falles (s. oben). Anf der einen Seite müssen wir also eine überaus regelmässige Wucherung der Randzone der secundären Augenblase annehmen, welche kein Hinderniss fand, so dass der Schliessungsprocess am distalen Pol vollständig zu Stande kam, auf der andern Seite aber einen gewaltigen Zerstörungs- and Aufsangungsprocess, welcher die Hindernisse, Linsenanlage und Mesodermaschicht, gänzlich verschwinden liess, so dass der Glaskörper sich direkt an die Retina anlegen konnte. Dazu kommt noch eine Erfahrungsthatsache. Alle jene Fälle nämlich von Aphakia congenita (es können natürlich nur die wenigen mikroskopisch untersuchten Falle in Betracht kommen), bei denen man mit Recht die Behauptung aufgestellt hat, dass die Linsenanlage nicht unbedingt von vorne herein als unterblieben zu betrachten sei, sowie jene Falle, bei denen wir noch den Zerstörungsprocess in Thätigkeit finden, also noch ein Linsenrudiment, berechtigen uns eben desshalb zu jener Annahme, weil sie den Stempel von Atrophie normaler Bestandtheile, Hypertrophie des Bindegewebes, Degeneration und Formbildungsanomalie tragen. Es ist mir kein Fall bekannt, wo die ubrigen Bestandtheile des Bulbus auch nur entfernt so entwickelt waren wie in meinem Fall. Man vergleiche die Falle von Helmholtz*), Manz**), Falchi ${ }^{*}$ ) und Michel $\dagger$ ), bei welchem jenes Knorpelstück nach Manz doch vielleicht ein Bulbusrudiment sein könne.

Gerade dieser Punkt ist für mich maassgebend, dass wir eine normale Retina und Pigmentschicht haben, dass ebenso die andern Hüllen keine degenerativen Processe auf-

*) No. 9 S. 269.

**) No. 20 S. 225 und No. 19 S. 161 .

** No. 4 .

†) No. $23 \mathrm{~S} .71$. 
weisen, sondern nur auf einem embryonalen Standpunkt zurückgeblieben sind; ein Process, der Linse und Linsenkapsel verschwinden liess, musste doch auch wohl in den andern Gebilden Spuren seiner zerstörenden Thätigkeit hinterlassen haben.

Eher könnte man noch geneigt sein, die Linsenbildung an einer abnormen Stelle, vielleicht an der ventralen Fläche der primären Augenblase, zu suchen; aber auch hier findet sich nichts, was eine derartige gezwungene Annahme rechtfertigen konnte. Ich betrachte also aus allen diesen Gründen die ectodermale Einstülpung als ausgeblieben.

Es entsteht nun eine weitere Frage: ist die Einstülpung der primären Augenblase am distalen Pol auch ausgeblieben? Diese lässt sich nach meiner Ansicht nicht mit Bestimmtheit entscheiden and ich werde versuchen, die Entstehung meiner Missbildung auf beide Arten zu erklären. Es wăre ja möglich, dass die Einstülpung der primären Augenblase auf ganz normale Weise vor sich gegangen wäre, ohne dass das Ectoderm mit folgte. Anzunehmen, dass das Ectoderm immer mit folgen müsse, dazu liegt gar kein zwingender Grund vor. Der zwischen dem Hormblatt und der sich einziehenden distalen Wand der primären Augenblase entstehende Raum konnte ja mit einer körperlymphartigen Flüssigkeit ausgefüllt werden. Die Bildung wäre also ganz analog der Linsenbildung beim Triton (s. Kessler's Fig. 56, Taf. IV). Dort sehen wir, wie nur die innere Zellenlage zur Linsenbildung verwandt wird, während die äussere Lage glatt über den sich bildenden Raum hinwegzieht. Die so sich ausbildende secundäre Augenblase unterlag nun aber in Bezug auf ihre Weiterentwicklung ganz andern Verbältnissen. Es fehlte ja nun am distalen Pol das formative Element, die Linse, welche vielleicht die einzige Ursache ist für die Pupillaröffnung; ' man kann sich ferner denken, dass die Faltung am Ciliarkörper und das Vorwachsen der Randzone der 
secundären Augenblase zu einer zweisehichtigen Zellenlage nur durch die Linse bewirkt wird. Feblt nun dieselbe, so verwächst schon früh die ganze Randzone wieder, ohne eine Spur davon zu hinterlassen. Bei einem Vorhandensein derselben wäre dies wenigstens in so regelmässiger Weise unmöglich gewesen. Analoge Processe finden sich ja in der Entwickelungsgeschichte viele; ich erinnere nur an die Abschnürung des Amnion, des Räckenmarks, der Gehörbläschen, der Linse ete. Die Glaskörperbildung erfolgte dann an der ventralen Fläche der gesammten Einstülpung an der sogenannten fötalen Augenspalte, welche ja noch vorhanden ist; derselbe füllte dann den ganzen Innenraum aus und legte sich überall der innern Lamelle an.

Ich muss hier bemerken, dass ich diese Erklärungsweise anfangs für weniger wahrscheinlich hielt, und dass erst eine Besprechung mit Herrn Professor Dr. Kupffer mir dieselbe wahrscheinlicher gemacht hat.

Andererseits wäre es nun möglich, dass an der Einstülpung der distale Pol nicht theilgenommen hätte, und dass sich dieselbe auf die ventrale Fläche der primären Augenblase beschränkt habe. Hier erfolgte dann die Glaskörperbildung; wie bei normalen Verhältnissen, und wir finden ja den Zusammenhang desselben mit dem Mesoderm im Colobom der Retina. Beide Annahmen haben etwas Positives für und gegen sich. Für letztere und gegen die erste spricht das Fehlen jeglicher Verwachsungsspur am distalen Pol; umgekehrt aber ist es doch höchst wunderbar, dass der distale Abschnitt der primären Augenblase, welcher unter normalen Verhältnissen ja eingestülpt und zur Retina wird, hier beim Ausbleiben der Einstülpang sich zu Pigmentepithel differencirt haben soll. Es wäre hier also die Frage zu entscheiden, ob die Zellen der primären Augenblase schon eine Eigenschaft besitzen, welche sie später zu Pigmentepithel- oder Retinazellen werden lässt, oder ob spätere Einflusse die Differencirung 
bedingen. Goette's *) Anschauung von der Zellenüberwanderung könnte uns hier vielleicht helfen, wenn wir eine etwas andere Richtung annebmen wollen.

Es sei mir hier gestattet, etwas näher auf die Anschauungen der Embryologen über die Einstüpungsursachen einzugehen. Ich habe diese Ansichten aus den betreffenden Werken kennen gelernt und bin daher nicht in der Lage, zu wissen, ob ihre Autoren sie noch obne alle Modification aufrecht erhaiten. Die Ansichten theilen sich ja dadurch, dass die einen die Ursache im Zellenleben selber suchen, andere dagegen mechanisehe Momente in Betracht ziehen. Die Frage, ob zwischen der Einstülpung des Ectoderms und der der primären Augenblase ein Zusammenhang vorhanden ist, ist noch unentschieden. Manz**) und Schwalbe vertreten die Anschaung, dass die ectodermale Einstülpung die der primären Augenblase zur Folge hat; Kessler †) nimmt fur beide dieselbe Ursache, die "Aspiration" von innen an. Goette $\dagger$ ) sucht die Ursache in "einer centrifugalen Zellenbewegung" nnd jener „radiär convergirenden Stosswirkung." Kölliker $\dagger \dagger$ ) endlich hält beide für zwei von einauder völlig unabhängige Vorgänge und sucht die Ursache allein in den ,Aeusserungen des Zellenlebens". Er beobachtete beim Kaninchen und Goette beim Bombinator, dass die Finstülpung der primären Augenblase etwas fruher beginnt als die des Ectoderms. Dasselbe fand Ciaccio*十) an Säugethier- und Vogelembryonen „auf das Evidenteste bewiesen”.

Meine Missbildung macht es uns nun in hohem Grade wahrscheinlich, dass die ectodermale Einstülpung in keinem

*) No. 5 S. 323 .

\%) No. 17 S. 7 .

***) No. 29 S. 78

†) No. 11 S. 33 .

t) No. 5 S. 323 .

t十t) No. 12 S. 628 .

将) No. 21 S. 73,1878 .

₹. Graefe's Arch"r fitr Ophthalmologie, XXXIV. 3. 
Zusammenhang mit der der primären Augenblase steht, jedenfalls nicht als ursächliches Moment aufgefasst werden kann. Sie lässt sich also nicht mit der Ansicht von Sehwalbe und Manz vereinigen und spricht sehr für die von Kölliker. Bei den von Kessler und Goette aufgestellten Ursachen könnte man sich in meinem Fall etwa denken, dass sie nicht zu genugender Wirkung kamen, weil die dazwischen liegende Mesodermalage zu dick war. Nach Angelucci*) ist dieselbe bei Säugern anfangs ziemlich dick und wird bis zum Beginn der Einstülpung immer dünner. Hier könnt man nun wohl mit Recht eine abnorm dicke Scheidewand als hemmende Crsache vermuthen. Die Lage des Bulbus hinten in der Orbita, der etwas kürzere Opticus deuten auf eine geringere Länge des Augenblasenstiels, mithin nach Angelucci auf eine dickere Mesodermazwischensubstanz. Erklären wir aber meine Missbildung auf die zweite Art, so treten beide Theorien in ein eigenthumliches Licht. Eine Ursache, wesshalb hier die ventrale Fläche eingezogen wird, wesshalb die centrifugale Zellenbewegung ihre Richtung ändert, lässt sich wohl sehwerlich vermuthen.

Ich glaube, dass die mechanischen Ursachen, welche wir uns bei den versehiedenen Einstülpungsprocessen denken können, immer nur in secundärer untergeordneter Weise in Betracht kommen, and dass Kolliker's Anschauung das wichtigste Moment trifft. Kolliker sagt: „Das Wachsthum von Organismen muss in erster Linie wesentlich aus dem Wachsthum ihrer Formbestandtheile abgeleitet werden". Ich habe alle die vortrefflichen Abbildungen, welche sich in den oben genannten Werken finden, mit einander verglichen, muss aber bekennen, dass ich die Art und Weise, wie Kölliker sich den Einstülpungsprocess vor sich gehend denkt, nicht verstehe, und glaube ferner,

*) No. 1 S. 1503 
dass man nicht umhin kann, auch auf die Umgebung Rücksicht zu nehmen. Wir können uns das etwa in der Weise denken, dass die auf „Aensserungen des Zellenlebens" beruhende Einstülpung in der Richtung stattfinden wird, wo der Widerstand am geringsten ist. Kolliker *) sagt: „Die Augenblase zieht sich ein, stülpt sich ein, wird eingestülpt, wenn am distalen $\mathrm{Pol}$ derselben ein Ruhepunkt eintritt, ringsum die Zellen energisch sich vermehren, so dass eine Flächenvergrösserung eintritt”. Kölliker beruft sich dabei auf die Gastrulabildung, „in welchem Falle sicherlich keine von aussen wirkende Momente vorhanden sind". Diesen Ruhepunkt nun kann ich nicht finden, und andererseits würde ein solcher, gleichsam Ueberwucherungsprocess, das Bild einer epibolischen Einziehung bieten, welches ganz anders aussieht, als die embolische Einsiz̈lpung. Alle Abbildungen zeigen mir aber, dass überall, wo solche Einstülpungsprocesse stattfinden, gerade in der Mitte derselben die grösste Dicke vorhanden ist, die meisten Zellen sich finden, dass wir demnach annehmen müssen, dass hier gerade die Zellvermehrung am grössten war. Besonders deutlich sehen wir dies z. B. in der Mitte der ectodermalen Einstülpung (siehe Kessler ${ }^{* *}$ ) Taf. I, Fig. 3-6, Taf. 17, Fig. 56, Taf. V, Fig. 66-67. Angelucci**) Taf. VII, Fig. 4, 8, 9 und andere Abbilduugen). Auch Goette $\dagger$ ) fand es so und sagt: „Sehr bald plattet sich ihre Aussenfläche nicht nur $a b$, sondern erscheint in der Mitte, wo sie am dicksten ist, nach innen gedrückt". Nehmen wir einmal den einfachsten Fall, die Gastrulabildung. Ich fand in Balfour's $\dagger_{\dagger}$ ) vergleichender Embryologie zwei Abbildungen neben einander, eine Blastula und

\footnotetext{
*) No. 12 S. 630 .

**) No. 11.

No. 1.

†) No. 5.

†) No. 34 .
} 
eine beginnende Gastrula. Hier sehen wir nun, dass an der Einstülpungsstelle eine Flächenvergrösserung stattgefunden hat, wir können uns den eingestülpten Theil der Wandung nicht mehr zu einer Blastula hinaus drückbar denken. Wir sehen aber an dem eingestülpten Theil äberall gleichmässig ausgebildete Zellen und keinen Rubepunkt. Es bedingte die energische Zellenvermehrung, welche local am Einstülpungspunkt der Blastula stattfand, eine Oberflächenvergrösserung, von welcher die notbwendige Folge eine Ausbuchtung sein musste. Dass diese Ausbuchtung nun nach innen stattfand, zeigt uns nur an, dass hier der geringere Widerstand vorhanden war. Bei der Gastrula sind es jene um die Einstülpungsstelle herumliegenden Zellen, welche einen festen Ring, eine feste Zone, um das nach Oberflächenvergrösserung strebende Kugelsegment bildeten, so dass dieses sich einbuchten musste; der Widerstand lag also in der Wandung der Umgebung selbst. Er kann nun aber auch in der Umbüllung liegen, dann bekommen wir eine Faltung. So vergrössert sich z. B. die secundäre Hirnblase sehr sehnell, während ihre Hülle es nicht thut; die Folge davon ist, dass das kugelige Wachsthum der Blase aufhört und Faltungen auftreten, als einzig möglicher Ausweg für die Flächenvergrősserung.

Noch eine Bemerkung über den Zusammenhang zwischen der Rinstulpung des Ectoderms und der der primären Augenblase. Die ectodermale Einstülpung wird uns dann als Ursache für die der primären Augenblase am distalen Pol erscheinen, wenn die locale stärkere Zellenvermehrung und die dadurch bewirkte Oberflächenvergrösserung im Ectoderm früher auftritt, als in der Wand der primären Augenblase; in diesem Falle mag auch dadurch am distalen Pol für die primäre Augenblase ein gewisser äusserer Widerstand geschaffen werden, welchen man als ursächliches Moment für die Finziehung derselben betrachten kann. Tritt nun aber, wie dies beim Kaninchen und Bombinator der Fall zu sein 
scheint, die local gesteigerte Zellenvermehrung und das Streben nach Oberflächenvergrösserung zuerst am distalen Pol der primären Augenblase auf, so zieht sich diese, di der Widerstand nach aussen offenbar ein grösserer ist, als nach innen, zuerst ein. Wir können demnach nicht die Linsenbildung als Ursache für die Bildung der secundären Augenblase auffassen, sondern müssen das Hauptgewicht auf die Thätigkeit der Zellen legen. In meinem Fall blieb offenbar die Vermehrungsthätigkeit der ectodermalen Zellen aus und damit auch jegliche Spur einer beginnenden ectodermalen Einstülpung und deren Resultat, die Linse. Dass das Fictoderm keine ganz normale Beschaffenheit hatte, seben wir auch daraus, dass die ganze Baut der Augengegend atrophisch, die Lider sehr klein sind. Mag immerhin als Hauptursache dafür der Mangel eines gesunden Bulbus angenommen werden, ich glaube, dass man auch daraus eine gewisse Schwäche des Ectoderms vermuthen kann.

Nehme ich meine erste Erklärungsweise an, so vollzog sich der Einstülpungsvorgang der primären Augenblase nach den soeben entwickelten Gesetzen. Bei meiner zweiten Erklärungsweise tritt ein Factor mehr in den Vordergrund, dessen Spuren wir auch jetzt noch erhalten sehen, es ist das mesodermale Gewebe, welches an der ventralen Fläche der primären Augenblase liegt. Wir können uns vorstellen, dass dieses colossal wucherte und die ganze ventrale $W$ and einstülpte.

Diese zieht sich ja auch bei normalen Verhältnissen mit ein, wenn auch nicht in so grosser Ausdehnung, und wir sehen hier, wie am distalen Pol, immer eine ausserordentlich starke Zellenvermehrung. Ich glaube nun, dass diese Wucherung und Einstülpung so intensiv vor sich ging, dass die übrige Wandung der primären Augenblase in eine gewisse Spannung versetzt wurde, so dass, wenn am distalen Pol die normale Zellenvermehrung und Oberflächen- 
vergrösserung stattfand, diese sich leichter als Kugelfäehe uber den distalen $\mathrm{P}_{0}$ l weiter ausdehnen konnte, als sich ihrerseits auch noch falten. Die Randzone der so entstandenen secundären Augenblase suchte dann zur Absehnürung des Glaskörperstiels (mesodermale Wucherung) vorzuwachsen; und zwar fand dies vom distalen Pol her in derselben Weise statt, wie unter normalen Verhältnissen am Pupillarrand, wir finden hier jene zweischichtige Lage von Zellen, wie am Ciliarkörper, siehe die mikroskopische Beschreibung. Gerade diese Art des Vorwachsens im Verein mit dem Fehlen jeglicher Nahtstelle am distalen Pol macht mir meine zweite Erklärungsweise sehr wahrscheinlich. Tch will noch hinzufügen, dass, wenn man geneigt ist, mechanischen Verhältnissen grossen Einfluss zuzusprechen, man sich vorstellen kann, dass bei dieser Art der ventralen Einstülpung die Spannung am distalen $\mathrm{Pol}$ so stark wurde, dass sie sich auf das Fctoderm auch noch geltend machen konnte und dasselbe an der Einstülpung verhindern.

Wie man die Sache sich aber auch denken mag, so viel steht für mich fest: eine Einstülpung der primären Augenblase hat stattgefunden, die des Ectoderms hat gefehlt. Jedenfalls hat ferner an der ventralen Fläche der Augenblase eine starke Wucherung des Mesoderms stattgefunden, das beweisen uns die noch jetzt vorhandene Verbindung des Glaskörpers mit dem Mesoderm und die Blutgefässreste im Colobombereich. Vermuthen können wir wegen des Linsenmangels endilich noch einen Schwächezustand der Vermehrungsfähigkeit der Ectodermazellen.

Ich gehe jetzt zu den Einzelheiten über, zunächst zur Glaskörperentstehung. Darüber, dass dieser nur von unten her durch den sogenannten fötalen Augenspalt in das Innere der secundären Augenblase gelangt ist, ist kein Zweifel möglich. Die im Glaskörper vorhandenen Zellen und Zellreste*)

*) No. 27 S. 157 und No. 12 S. 613 a. 645. 
deuten wohl auf seine mesodermale Entstelung hin. Das scheint mir wenigstens aus meinem Fall mit hoher Wahrscheinlichkeit hervorzugehen, dass jene mit der Linsenbildung: unter normalen Verhältnissen eingestülpte Mesodermaschicht nichts mit der Glaskörpergenesis za schaffen hat; denn es ist ja ein Glaskörper vorhanden, ohne dass jene Schicht mit eingestülpt worden ist. Arnold, Lieberkühn und Michalkovicz vertreten, wie ich aus Löwe's*) Abhandlung ersehe, diese Anschauung, ebenso Angelucei. $* *)$

Die zu solid gewordenen Strängen obliterirten Gefässreste im Glaskörper zeigen uns noch den Uebergang der Glaskörpergefässe in die Retinalgefässe an. Es ist dies ja etwas ganz normales, wenn man die ron H. Müller***) zuerst aufgestellte Auschauung annimmt, dass die Vascularisation der Retina vom Glaskörper ausgeht. Man vergleiche auch die Erfahrungen von Eversbusch $\dagger$ ) und Löwe. $\dagger$ )

Meine Begrenzungsmembran des Glaskörpers, die Hyaloidea, ist bei der Schrumpfung desselben nie an der Retina, sondern immer am Glaskorper haften geblieben und geht im Colobombereich in das mesodermale Gewebe uber. Sie scheint mir also dem Glaskörper anzugehören und auch wie dieses vom Mesoderm abzustammen, wie es Schwalbe $\dagger+$ ) und Lieberkühn ${ }_{\dagger}$ ) ja auch annehmen. Eine Limitans interna retinae, welche Kölliker*⿻三丨 eine Hyaloidea, konnte ich in beiden Augen nicht entdecken. Fast immer gelang es mir, jene rundlichen endothelartigen Gebilde, an denen nur selten ein Kern wahrzunehmen war,

*) No. 16 S. 545 .

***; No. 1 S. 154.

***) No. 24 .

†) No. 3 S. 40-50.

t) No. 16 S. 547 .

tit) No. 29 S. 136 und No. 30 S. 438 .

*) No. 15 .

**t) No. 12 s. 123-125. 
auf der Flächenansicht (an Stellen, wo die Hyaloidea sich losgelosst hatte und ungeklappt war) zu erkennen; nur dort, wo die Membran im Bereich des Colobom der Retina in das mesodermale Gewebe übergeht, ist sie meistens structurlos, und nur an einigen Stellen sah ich jene rundlichen Gebilde deatlich. Herzog Carl Theodor*) spricht ja, wie bekannt, dem Glaskörper eine eigene Umbüllungsmembran ab. Er fand die Oberfläche seiner Limitans, welche dem Glaskörper zugewendet ist, immer glatt und im normalen Auge konnte er kein Endothel auf ihr nachweisen. Wohl aber fand er seine Limitans bedeckt mit einem einschichtigen Endothelzellenlager, in einigen Fällen von Glaskörperablosung. Es ist ja möglich, dass im Leben bald die endothelartigen Zellen gänzlich versehwinden und dass sie in den Eällen von Glaskorperablosung in Folge der Alteration wieder entstanden sind. Hannover **) beschreibt auf seiner Limitans interna retinae eine einfache Epithelzellenlage an ihrer inneren Fläche. Ob dies wohl dieselben Zellen sind, wie die meinigen? Herzog Carl Theodor verwahrt sich dagegen, dass seine Endothelzellen etwa jene Hannover'schen Epithelzellen sein könnten, ohne aber die näheren Gründe dafür anzugeben.

Die mannichfachen Faltungen der Retina in beiden Augen beruhen, wie ich schon oben gesagt habe, wohl allein darauf, dass die innere schnellwachsende Lamelle den Raum nicht vorfand, welchen sie brauchte, um sich in kugeliger Form auszudehnen. Je grösser die Raumbeschränkung ist, desto mannigfaltiger werden die Faltungen, desto schwerer entwirrbar. Am schwersten gestalten sich diese Verhältnisse innerhalb des Pigmenteoloboms. Woher kommt jenes wirre Durcheinander der Retinalschichten? Auch hier glaube ich, dass es nur auf eine Faltung aus Mangel an Raum

*) No. 2.

**) No. 2 S. 121. 
beruht, und die Verhältnisse nur deshalb complicirter sind, weil wir annehmen müssen, dass sich bei überwiegendem Wachsthum der inneren Lamelle der secundären Augenblase sich dieselbe in sehr früher Zeit zuerst über die änssere hinansgeschoben hat, so dass die Umschlagstelle in die innere Lamelle selber zu liegen kam, mithin dieselbe also im Colobombereich des Pigmentepithels doppelt und zwar za in einander umgekehrter Lage vorhanden war. Ich habe darauf oben besonders aufmerksam gemacht (s. Fig. 4). So kommt es, dass wir innerhalb des Pigmentcoloboms noch Retina haben. Die äussere Lamelle hat damit nichts zu thun, sie hört am Colobomrand der Pigmentepithelschicht auf; höchstens kann man noch jene platten unpigmentirten Zellen zu ihr rechnen (s. Fig. 4c). Haab*) befand sich daher wohl im Irrthum, als er glaubte, dass die äussere Lamelle auch Bausteine zur Retina geliefert habe, Haab sagt: „Nachdem sich die äussere und innere Lamelle vereinigt, nimmt die Retina die oben erwähnte, höchst unregelmässige Structur an." Das könnte man auch in meinem Fall denken. Das aufmerksame Verfolgen der Retinalbestandtheile an dieser Stelle auf Serienschnitten hat mich jedoch zu einer anderen Ansicht kommen lassen, welche ich oben auseinandergesetzt habe. Die Hauptsache dabei ist die körperliche Vorstellung, welche man aus einer Serie von Schnitten immer gewinnen kann, aber nie aus einem einzelnen Schnitt allein. Alles Retinalgewebe zwischen g, Fig. 4 und 5 und dem Sehnerveneintritt stammt nur von der inneren Lamelle ab. Den auf Fig. 5 von $g$ aus nach etwas oben und dann lateralwärts verlaufenden Colobomrand des Pigmentepithels haben wir als die obere Grenze des ursprünglichen Randes der secundären Augenblase aufzufassen. Hier von oben fand das Verwachsen der inneren Lamelle am stärksten statt, sodass sie zuletzt bis zur

*) No. 8 S. 262-264. 
gezackten Linie auf Fig. 5 reichte. Die Umschlagstelle lag bei diesem Process in der inneren Lamelle selbst und wir müssen, wie es thatsächlich der Fall ist, eine doppelte Retina haben, welche aber zu einander in umgekehrter Lage liegt. Da nun diese Wachsthumsausdebnung der inneren Lamelle vermuthlich schon in sehr fruher Zeit stattfand, die sich varschiebende Lamelle aber ein Hinderniss fand, den Glaskörperstiel, welchen sie nun abzuschnüren versuchte, so ist es sehr wahrscheinlich, dass bei diesem Vorschieben, wobei die Umschlagstelle immer wechselte, and dem Andrängen an den Glaskörperstiel eine Verschiebung ihrer einzelnen Elemente stattfand, woraus sich vielleicht jenes anscheinend wirre Durcheinander erklären lässt. Wie wir sehen, findet sich die grösste Unorduung in den äusseren Schichten, was bei der Art der Verschiebung ganz natürlich ist. Ich muss noch darauf aufmerksam machen, dass dieses Vorschieben der inneren Lamelle von oben nicht auf einer localen Wucherung ihrer Randzone beruht, sondern auf das Wachsthum der ganzen, die obere Halbkugel auskleidenden inneren Lamelle zu beziehen ist, daher finden wir auch nur eine kleine Falte der Retina im oberen $\mathrm{Ab}$ schnitt. Ganz dieselben Verbältnisse finden wir im lateralen Abschnitt und am lateralen Colobomrand. Interessant ist es nun, dass wir, weil die innere Lamelle sich am medialen und unteren Rand des Pigmentcoloboms (s. Fig. 5) zwischen $g$ und $d$ nur sehr wenig and zwischen $d$ und $f$ gar nicht vorschob, dass wir nun im unteren medialen vorderen Quadranten Faltungen der Retina finden, und zwar darunter eine sehr bedeutende.

Ganz charakteristisch finde ich auch die Faltungen im rechten Auge. Einmal liegt eine Hauptfalte an der Ora, also an einer Stelle, wo ursprünglich die Umschlagsstelle der Lamellen der secundären Augenblase am distalen Abschnitt lag; erst später wächst sie ja hier zur Bildung des Corpus ciliare und der Iris vor. Es ist dasselbe Bild, wie 
an der unteren medialen Seite des Coloboms im Mikrophthalmus (Fig. 5 d-f siehe die mikroskopische Beschreibung). Zweitens jener Faltungsbezirk an Stelle der zu erwartenden Macula lutea, welcher meiner Ansicht nach unmogglich (den Grund s. oben) als Folge von Schrumpfung bei der Härtung angesehen werden kann.

Auch hier, wo nach Schwalbe früher die fötale Augenspalte vorhanden war, an der Umsehlagstelle der Lamellen der secundären Augenblase, entsteht wieder die Faltung. Auf die Ursachen, weshalb diese Hauptfaltungen gerade an der Umschlagsstelle auftreten, will ich hier nicht weiter eingehen; ich will noch hinzufügen, dass ich in einem Präparat ron doppelseitigen Mikrophthalmus, gerade an diesen Stellen wieder die Hauptfaltungen der Retina fand. Das Resultat der Untersuchung werde ich später vielleicht veröffentlichen.

Manz*) und andere Autoren haben den Narbenzug zur Erklärung jener wirren Schichtung herbeigezogen. In meinem Fall ist jedenfalls kein Narbengewebe vorhanden. Thalberg**) suchte nach der von Deutschmann zuerst aufgestellten Ansicht in seinem Fall von Colobom die Genese desselben in einer intranterinen Entzündung. Meine Missbildung zeigt nur normales Gewebe, gut entwickeltes und embryonales.

Auch das Colobom der Retina mit grossem äquatorialen Durchmesser and kurzem meridionalen lässt sich aus Wachsthumsvorgängen sehr gut erklären. Denken wir uns die Glaskörpereinstülpung (also die fötale Augenspalte) auf breiter Basis erfolgt und das zum Schluss führende Wachsthum der Retina senkrecht zur Länge derselben fortschreitend, so bleibt schliesslich als Rest ein Colobom von der Breite der Spalte. Es kommt ausserdem der immer

*) No. 21 .

No. 32 S. 81 . 
stärker werdende intraoculare Druck dabei in Betracht und die Resistenz des Glaskörperstiels bei der Abschnurung. Dass die sogenannte fötale Augenspalte in allen Fällen, in denen Colobom in den Lamellen der secundären Augenblase sich vorfindet, ursprünglich in fötaler whene breiter als unter normalen Verhältnissen ist, scheint mir sehr wahrscheinlich. Ebenso kann man die versehiedenen meridionalen Lagen des Coloboms derselben auf âhnliche Wachsthumsvorgänge zurückzuführen versuchen, wobei nicht ausgeschlossen bleibt, wie weit eine eventuelle Rotation des Bulbus dabei mitwirkt. Meistens wird diese nach meiner Ansicht, wenn der Glaskörperstiel persistirt (obliterirte Blatgefässe) wobl wenig in Betracht kommen. In meinem Fall müssen wir sie wohl als ausgeblieben betrachten. Erstens, weil wir das Colobom, s. Fig. 5, im hinteren unteren inneren Quadranten haben, und zweitens, weil der Opticus in einer Richtung von oben medial nach unten lateral in den Mikrophthalmus eintritt und zwar genan am proximalen Pol, anstatt etwas medianwarts von ihm. Denken wir uns nun, dass, wie es ja bei normalen Verhältnissen der Fall sein muss, wenn man überhaupt eine Rotation annehmen will, dass ohne Störung sich eine untere grössere Bulbushalfte ausgebildet und nun eine fast $1 / 4$ Rotation stattgefunden hätte, so tritt der Opticus in normaler Richtung ein und der hintere Augenpol liegt lateral von ihm.

Schwieriger zu erklären ist das Chorioideal-Colobom und die Verdünnung der Sclera. An einen intraocularen Druck kann man wohl kaum als Ursache denken, welcher zur Zeit, two dies entstand, wohl sehr gering war. Die Schliessung der fötalen Augenspalte fällt ja, wie bekannt, in eine sehr frühe Zeit, Ende des zweiten Monats. $\mathrm{Zn}$ dieser Zeit sind aber die Sclera und Chorioidea noch nicht ausgebildet und können nicht als feste Umbüllungsmembranen gedacht werden. Es liegt nun das Chorioideal-Colobom und die Stelle der grössten Verdünnung der Selera gerade 
dort, wo die Retina wegen ihrer doppelten Lage am dicksten ist (s. Fig. 5 und Fig. 4). Ich glaube nun, dass die Verdickung der inneren Lamelle, ihre Verdoppelung, vielleicht als mechanisches Moment aufgefasst werden kann. Die Ausdehnungsfähigkeit derselben schaffte sich hier den Raum auf Kosten des Mesoderms, ,die Aeusserungen des Zellenlebens waren auch hier wieder die Ursache." - Das Ausbleiben der ectodermalen Finstülpung verursachte das Fehlen der vorderen Augenkammer, das Zusammenwachsen der Ciliarmuskelfasern etc.; ebenso auch die Abplattung des ganzen Mikrophthalmus in sagittaler Richtung. Die mangelhafte Differenzirung in der Tunica externa und media beruht auf einer Entwicklungsstörung, deren Ursachen wir wohl kaum feststellen können. Wir können nur eine Ernährungsstörung vermuthen, welche vielleicht auf Blutgefässanomalien beruht.

In Betreff der Phylogenese endlich verweise ich auf die Myxine, deren Auge W. Müller*) genauer untersucht hat. Hier haben wir auch eine secundäre Blase mit einer Oeffnung, durch welche ein Gefäss eintritt, umhüllt von Sebleimgewebe und im Innern der Blase einen ,Schleimgewebsstock, welcher der Glaskörperanlage der höhern Wirbelthiere entspricht". Vergleiche die Abhandlung von Müller.

Der Befund an den Optici und Tractus ist mir ganz unklar. Bei vollständiger Kreuzung (Michel) müsste der rechte Tractus viel dünner sein als der linke; ebenso, wenn man annimmt, dass sich das grössere Bündel kreuzt, (Gudden**) und Purtscher***). Dei linke Tractus ist kleiner, besitzt aber nur normale Nervenfasern und keine Rosafürbung; der rechte ist dicker, zeigt verwaschene Nervenfasercontouren und Rosafärbung. In den Optici sind

*) No. 25.

**) No. 7 S. 1 u. 237.

\%*) No. 28 S. 191. 
die Nervenfasercontouren ziemlich gleichmässig scharf, im Ganzen sind die Fasern links etwas kleiner und die Rosafärbung ist links in geringem Grade rorhanden. Man könnte vielleicht annehmen, dass der ganze linke Tractus in den rechten Opticus übergeht und kein ungekreuztes Bündel vorhanden ist, der rechte Tractus grösstentbeils in den linken Optieus und sein ungelreuztes Bündel im rechten Opticus nur nicht zu erkennen ist. Die Verhältnisse sind unklar. Der Umstand aber, dass die Atrophie im rechten Tractus grösser ist als im linken Opticus, lasst uns eine centrale absteigende Störung vermuthen.

Es sei mir zum Sehluss gestattet, meinem hochverehrten Lehrer, Herrn Professor Dr. v. Rothmund, für die Deberweisung dieser Arbeit meinen besten Dank auszusprechen und ebenso ihm und dem Herrn Professor Dr. Kupffer und Privatdocent Dr. Sehloesser für die Güte, mit der sie meine Präparate durchsahen und mir meinen mikroskopischen Befund bestätigten. 
IV. Litteratur - Verzeichniss.

1. Angelueci, A., Ueber Fntwicklung und Ban des vorderen Uvealtractus der Vertebraten. Im Arch. f. mikrosk. Anat. XIX.

2. Carl Theodor, Herzog, Beiträge zur Anatomie und Pathologie des Glaskörpers. Im Arch. f. Ophth. XXV. 3.

3. Eversbusch, 0., Klinisch-anatomische Beiträge zur Embryologie und Teratologie des Glaskörpers. In Mittheilungen aus der Kgl. Augenklinik zu München, 1882.

4. Falchi, Mikrophthalmus congenitus. $1884 \mathrm{Ann}$. di Ottalm.

5. Goette, Alex, Die Entwicklungegeschichte der Unke als Grundlage einer vergleichenden Morphologie der Wirbelthiere. Leipzig 1875.

6. Gudden, v., Experimental-Untersuchungen über das Scbädelwachsthum. München 1874.

7. _ - Ueber Kreuzung der Nervenfasern im Chiasma. Im Arch. f. Ophth. XXV. 3 च. 4.

8. Haab, O., Beiträge zn den angeborenen Fehlern des Auges. Im Arch. f. Ophth. XXIV. 2.

9. Helmholtz, Mikrophthalmus. In Graefe's Archiv III. 2.

10. Joseph, G., Ueber Gestaltungen der knöchernen Augenhöhle nach Verlust des Augapfels. Referat in Michel's Jahresberichten, 1877.

11. Kessler, L., Entwicklungsgeschichte des Auges der Wirbelthiere. Leipzig 1877.

12. Kölliker, A., Entwicklungsgeschichte des Menschen und der höhern Thiere. Leipzig 1879.

13. - - Grundriss der Entwicklungsgeschichte des Menschen und der höhern Thiere. Leipzig 1884.

14. - - Zur Entwicklung des Auges und des Geruchsorganes menschlicher Embryonen. Würzburg 1883.

15. Lieberkühn, Ueber das Auge des Wirbelthierembryo. 1872. 
16. Loewe, L., Beiträge zur Anatomie des Auges. Arch. f. mikrosk. Anat. XV.

17. Manz, W., Entwieklungsgeschichte des menschlichen Auges. In Graefe-Saemisch's Handbuch der gesammten Augenheikunde II, Cap. V.

18. - - Missbildungen des menschlichen Auges. In Graefe-Sämisch's Hdb. der gesammten Augenheilkunde II, Cap. VI. Leipzig 1876.

19. - - Z Zwei Falle von Mikrophthalmus cong. Graefe's Arch. XXYI. 1.

20. - - Kryptophthalmus. In Zehender's klin. Monatsbl., 1872.

21. - - Referate über Augenmissbildungen in Wichel's Jahresberichten.

22. Magnus, Drei Falle von Mikrophthalmus. Arch. f. Ophth. XII. S. 297.

23. Michel, Anophthalmus cong. Arch. f. Ophth. XXIV. 2.

24. Müller, H. Würzburger naturwissenschaftil. Zeitschrift, Bd. II.

25. Müller, W., Ueber die Stammesentwicklung des Sehorgans der Wirbelthiere. Leipzig 1875.

26. Pause, Anatomischer Befund beim Colobom der Iris und Chorioidea. Arch. f. Ophth. XXV.

27. Potechin, Ueber die Zellen des Glaskörpers. Virchow's Arch. f. path. Anatomie 72.

28. Purtscher, 0., Ueber Kreuzung und Atrophie der Nerri et Tractus optici. Arch. f. Ophth. XXV. 3.

29. Schenkl, Monophthalmus cong. Prager ned. Wochenschrift No. 48, 1883.

30. Schwalbe, G., Lehrbuch der Anatomie der Sinnesorgane Erlangen 1883.

31. - - Mikroskopische Anatomie des Sehnerven, der Netzhaut und des Glaskörpers. Graefe-Sämisch's Handbuch der gesammten Augenheilliunde I, Cap. IV. Leipzig 1874.

32. Stöhr. Ph.. Histologie und histologische Technik. 1887.

33. Thalberg, Zur pathologiseben Anatomie des Coloboms der Chorioidea und Iris. Graefe's Arch. XIII. 1.

34. Vetter, Cobersetzung der vergleichenden Embryologie von Fr. Balfour. 


\section{Figuren - Erklärung.}

Fig. 1. Horizontaler Meridionalschnitt durch den Mikrophthalmus.

a. Corneascleralgrenze.

b Aufhören des Pigments.

c Glaskörper.

d Lamina cribrosa.

e Chorioidea.

f Cornealepithel.

g Sichel von Ciliarkörpergewebe.

h Retina (kleine Falte).

i Stelle der wirren Schichtung.

$\mathrm{k}$ Faltenende einer Retinalfalte.

Fig. 2. Horizontalschnitt, Stück desselben aus dem bintern untern Quadranten in der Höhe von a und b auf Fig. 5 .

a Mesodermales Gewebe und Retinalcolobom.

b ebenso.

c Anfhören der Pigmentirung.

d innere granulirte Sehicht.

Fig. 3. Frontalschnitte.

a durch die Optici $5 \mathrm{~mm}$ binter dem Bulbus. I linker, II rechter Opticus.

b durch die Optici dicht vor dem Chiasma. I linker, II rechter.

c durch die Tractus. I linker, II rechter.

C M Commissura Meineri.

Fig. 4. Horizontalschnitt, Stück desselben medial vom Sehnerveneintritt in der Höhe von $g$ anf Fig. 5.

a innere granulirte Schicht.

b platte pigmentlose Zellen.

c äussere Körnerschicht.

d Sclera.

e Chorioidea.

7. Graefe's Archiv für Ophthalmologie, XXXIV. 3. 
I Ciliargefäss.

g Punkt der Vereinigung der äusseren und imneren Lamelle.

h Nervenfasern.

Fig. 5. Schematische Darstellung der Colobome.

o Opticus.

- Colobom des Pigmentepithels.

.. $\quad$ der Chorioidea.

$\sim, \quad$ Retina. 

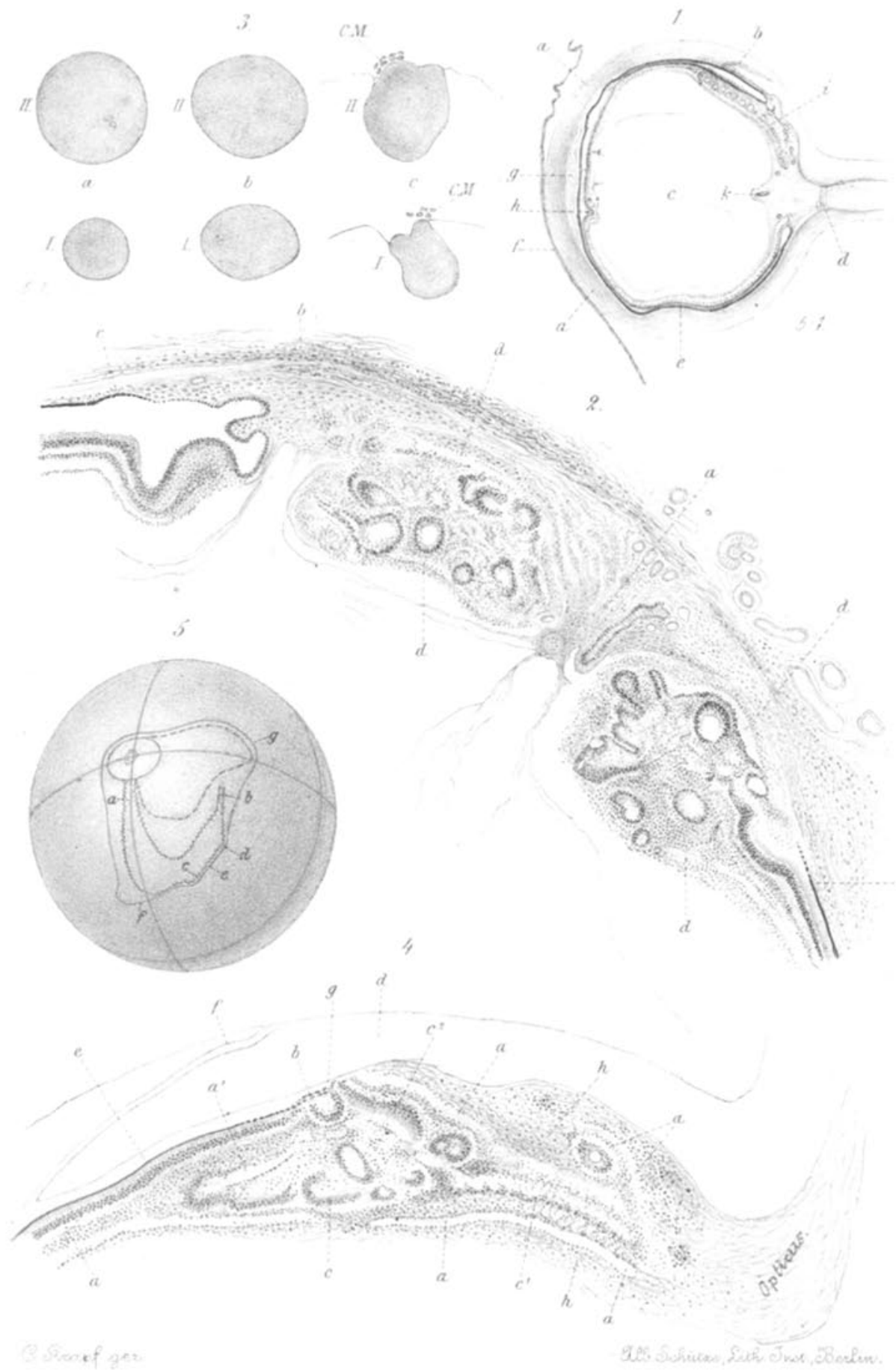